\title{
A Note on the First Rigid Cohomology Group for Geometrically Unibranch Varieties
}

\author{
Nobuo Tsuzuki \\ To Professor Francesco Baldassarri on the occasion of his $60^{\text {th }}$ birthday, \\ with much gratitude.
}

ABSTRACT - We prove a vanishing of the first rigid cohomology group for geometrically unibranch varieties with supports in a proper closed subset and apply it to the full faithfulness problem of the restriction functors of overconvergent isocrystals. As an application, we prove that the first rigid cohomology group is pure of weight 1 for proper and geometrically unibranch varieties over a finite field. We also establish a comparison result of rigid cohomology groups between a geometrically unibranch variety and its normalization.

\section{Introduction}

By the recent development one can understand the rigid cohomology for smooth varieties well. However, it is still difficult to understand it for singular varieties. In this note we study the rigid cohomology for mildly singular varieties. We prove a vanishing of rigid cohomology groups of degree $\leq 1$ for geometrically unibranch varieties with supports in a proper closed subset and apply it to the full faithfulness problem of the restriction functors of overconvergent isocrystals (see the full faithfulness problem in [31, Conjecture 1.2.1]). We calculate weights of Frobenius eigenvalues of the first rigid cohomology groups for geometrically unibranch varieties over finite fields. We also establish a com-

(*) Indirizzo dell'A.: Mathematical Institute, Tohoku University, Aramaki Aza-Aoba 6-3, Aobaku Sendai 980-8578, Japan.

E-mail : tsuzuki@math.tohoku.ac.jp

Partly supported by Grant-in-Aid for research (B) 22340001, the Ministry of Education, Culture, Sports, Science and Technology, Japan. 
parison theorem of rigid cohomology groups between a geometrically unibranch variety and its normalization, more generally for universal homeomorphisms. These results are $p$-adic analogues of the comparison theorem of inverse images for universal homeomorphisms in etale cohomology [1, VIII, 1].

\section{$1.1-$ Results}

First we recall the definition of the notion "geometrically unibranch". Let us fix notation. For a scheme $T$ over a spectrum Spec $k$ of a field $k$ and an extension $l$ of $k$, we put $T_{l}=T \times_{\text {Spec } k}$ Spec $l$. For a scheme $T, T_{\text {red }}$ (resp. $T_{\text {nor }}$ ) denotes the reduced closed subscheme associated to $T$ (resp. the normalization of disjoint sum of the reduced closed subschemes of irreducible components of $T$ in the total function field). We use the same notation $R_{\text {red }}$ and $R_{\text {nor }}$ for the associated rings of a ring $R$ as above.

A scheme $T$ is said to be geometrically unibranch if, for any point $t$ of $T$, (a) $\mathcal{O}_{T_{\text {red }}, t}$ is integral, (b) the normalization $\left(\mathcal{O}_{T_{\text {red }}, t}\right)_{\text {nor }}$ of $\mathcal{O}_{T_{\text {red }}, t}$ is local, and (c) the residue field of $\left(\mathcal{O}_{T_{\text {red }}, t}\right)_{\text {nor }}$ is a purely inseparable extension of the residue field of $\mathcal{O}_{T_{\text {red },}, t}[19,0,23.2 .1]$. If $T$ is a normal scheme over Spec $k$ for a field $k$, then $T_{l}$ is geometrically unibranch for any field extension $l$ of $k$ [19, IV, Proposition 6.15.6]. Moreover, if $T$ is geometrically unibranch, then the canonical morphism $T_{\text {nor }} \rightarrow T$ is a universal homeomorphism, that is, the morphism of associated topological spaces of any base change is a homeomorphism [19, IV, Definition 2.4.2, Corollaire 18.12.11].

Let $K$ be a complete discrete valuation field of mixed characteristics $(0, p), \mathcal{V}$ the integer ring of $K$, and $k$ the residue field of $\mathcal{V}$. Let $j_{X}: X \rightarrow \bar{X}$ be an open immersion of separated schemes locally of finite type over Spec $k$ (note that $\bar{X}$ might not be proper), $Z$ a closed $k$-subscheme of $X$, $U=X \backslash Z$ with the open immersion $j_{U}: U \rightarrow \bar{X}$, and $M$ a convergent isocrystal $M$ on $X / K$ overconvergent along $\bar{X} \backslash X$ (for simplicity, we say an overconvergent isocrystal on $(X, \bar{X}) / K$ later). We denote the rigid cohomology for an overconvergent isocrystal $M$ on $(X, \bar{X}) / K$ with supports in $Z$ by $H_{\operatorname{rig} Z}^{r}((X, \bar{X}) / K, M)$ (resp. $H_{\operatorname{rig} Z}^{r}(X / K, M)$ if $\bar{X}$ is proper), whose definition is recalled briefly in section 2.1. Then there is a canonical long exact sequence

$$
\begin{aligned}
& \cdots \rightarrow H_{\text {rig } Z}^{r}((X, \bar{X}) / K, M) \rightarrow H_{\text {rig }}^{r}((X, \bar{X}) / K, M) \rightarrow H_{\text {rig }}^{r}\left((U, \bar{X}) / K, j_{U}^{\dagger} M\right) \\
& \rightarrow H_{\operatorname{rig} Z}^{r+1}((X, \bar{X}) / K, M) \rightarrow \quad \cdots,
\end{aligned}
$$


which is called an excision sequence [7, Proposition 2.5]. One of the main results of this note is the following vanishing of rigid cohomology groups. The vanishing in the smooth case is known at least if $\bar{X}$ is proper over Spec $k$ [7, Théorème 3.8] [30, Theorem 4.1.1] (see the detail for a general $\bar{X}$ in section 2.2).

THEOREM 1.1. (1) If $U$ has a nonempty intersection with each connected component of $X$, then $H_{\operatorname{rig} Z}^{0}((X, \bar{X}) / K, M)=0$.

(2) Let $X=\cup_{\lambda} X_{\lambda}$ be the irreducible decomposition of $X$, and put $X_{\lambda, \mu}=X_{\lambda} \cap X_{\mu}, U_{\lambda}=U \cap X_{\lambda}$ and $U_{\lambda, \mu}=U \cap X_{\lambda, \mu}$. Suppose that

(i) $U_{\lambda}$ is dense in $X_{\lambda}$ for any $\lambda$,

(ii) $U_{\lambda, \mu}$ is dense in $X_{\lambda, \mu}$ for any $\lambda$ and $\mu$ if $X_{\lambda, \mu} \neq \emptyset$,

(iii) $X_{\lambda}$ is geometrically unibranch for any $\lambda$.

Then the vanishing $H_{\mathrm{rig} Z}^{1}((X, \bar{X}) / K, M)=0$ holds.

Note that each of the three conditions (i)-(iii) in the Theorem 1.1 (2) above is preserved by the base extension $X_{l}$ for any extension $l$ over $k$ (see [19, IV, Proposition 6.15.7] for the condition (iii)). We prove the theorem above for a smooth $X$ (to be self-contained) in Theorem 2.9 and for a general $X$ in sections 3 and 4 .

By the excision sequence above and Theorem 2.13 and Corollary 2.14 we have

COROLlaRY 1.2. With the same hypotheses of Theorem 1.1 (2), the restriction map

$$
H_{\text {rig }}^{0}((X, \bar{X}) / K, M) \rightarrow H_{\text {rig }}^{0}\left((U, \bar{X}) / K, j_{U}^{\dagger} M\right)
$$

is bijective, and the restriction map

$$
H_{\text {rig }}^{1}((X, \bar{X}) / K, M) \rightarrow H_{\text {rig }}^{1}\left((U, \bar{X}) / K, j_{U}^{\dagger} M\right)
$$

is injective. In particular, the restriction functor from the category of overconvergent isocrystals on $(X, \bar{X}) / K$ to the category of overconvergent isocrystals on $(U, \bar{X}) / K$ is fully faithful.

COROLlary 1.3. With the same hypotheses of Theorem 1.1 (2), suppose that $K$ admits a lift $\sigma$ of Frobenius. Then the restriction functor from the category of overconvergent $F$-isocrystals on $(X, \bar{X}) / K$ with respect to $\sigma$ to the category of overconvergent $F$-isocrystals on $(U, \bar{X}) / K$ with respect to $\sigma$ is fully faithful. 
When $X$ is smooth over Spec $k$, the full faithfulness results in Corollaries 1.2 and 1.3 were proved by a local study of $p$-adic differential equations by K. Kedlaya in [21, Theorem 5.2.2] (see also [31, Theorem 4.1.1]).

In etale cohomology, the similar property of Corollary 1.2 for a normal scheme $X$ follows from the surjectivity of the natural homomorphism $\pi_{1}^{\mathrm{alg}}(U) \rightarrow \pi_{1}^{\mathrm{alg}}(X)$ of algebraic fundamental groups. The following theorem is a $p$-adic analogue of the comparison of inverse images for universal homeomorphisms in etale cohomology [1, VIII, Corollaire 1.2, Examples 1.3 (c)].

THEOREM 1.4. With the notation as above, suppose $X$ is geometrically unibranch. Let $g: X_{\text {nor }} \rightarrow X$ be the canonical structure morphism, $\overline{X_{\text {nor }}} a$ compactification of $X_{\text {nor }}$ over $\bar{X}$ (take the normalization of $\bar{X}$, for example), $W$ be the inverse image of $Z$ in $X_{\text {nor, }}$, and $g^{*} M$ the inverse image of $M$ as an overconvergent isocrystal on $\left(X_{\text {nor }}, \overline{X_{\text {nor }}}\right) / K$. Then the natural homomorphism

$$
g^{*}: H_{\text {rig } Z}^{r}((X, \bar{X}) / K, M) \rightarrow H_{\text {rig } W}^{r}\left(\left(X_{\text {nor }}, \overline{X_{\text {nor }}}\right) / K, g^{*} M\right)
$$

is an isomorphism for any $r$.

As an application, we have a following weight result for geometrically unibranch varieties. The author ignores the corresponding result in the $l$-adic theory in literatures.

THEOREM 1.5. Suppose $k$ is a finite field of $p^{s}$ elements and that $K$ admits a lift $\sigma$ of Frobenius such that $\sigma^{s}=\mathrm{id}_{K}$. If $X$ is proper and geometrically unibranch over Spec $k$, then $H_{\mathrm{rig}}^{1}(X / K)$ is pure of weight 1 . Here $H_{\text {rig }}^{r}(X / K)$ denotes the rigid cohomology of $X / K$ for the unit convergent $F$ isocrystal $\mathcal{O}_{] X[}$ on $X / K$.

\section{2 - Ideas and plan}

In section 2 we recall some results on rigid cohomology, cohomological descent for proper hypercoverings [13] [32] [33] (see Propositions 2.5, 2.6 and Theorem 2.7), and alterations. Especially, the long exact sequence

$$
\begin{aligned}
0 & \rightarrow H_{\operatorname{rig} Z}^{0}((X, \bar{X}) / K, M) \rightarrow H_{\text {rig } W_{0}}^{0}\left(\left(Y_{0}, \bar{Y}_{0}\right) / K, M_{0}\right) \rightarrow \operatorname{ker}\left(d_{1}^{1,0}\right) \\
& \rightarrow H_{\operatorname{rig} Z}^{1}((X, \bar{X}) / K, M) \rightarrow H_{\text {rig } W_{0}}^{1}\left(\left(Y_{0}, \bar{Y}_{0}\right) / K, M_{0}\right)
\end{aligned}
$$


where $\quad d_{1}^{1,0}: H_{\text {rig } W_{1}}^{0}\left(\left(Y_{1}, \overline{Y_{1}}\right) / K, M_{1}\right) \rightarrow H_{\text {rig } W_{2}}^{0}\left(\left(Y_{2}, \bar{Y}_{2}\right) / K, M_{2}\right), \quad$ induced from the spectral sequence with respect to a proper hypercovering $\left(Y_{\bullet}, \bar{Y}_{\bullet}\right) \rightarrow(X, \bar{X})$ in Corollary 2.8 is one of the important tools we are going to use (see the notation in Theorem 2.7).

In section 3 we study the connectedness of tubes for connected schemes. The connectedness in Lemma 3.1 plays an essential role to prove Theorem 1.1. We also show the invariance of rigid cohomology groups of degree 0 for proper surjective morphisms whose geometric fibers are connected in Theorem 3.6. This is one of the keys of this note.

In section 4 we prove Theorem 1.1 (2). In order to prove it we take a regular alteration $\bar{Y} \rightarrow \bar{X}$ (Theorem 2.15) and consider Stein factorization of $\bar{Y} \rightarrow \bar{X}$. Starting from a smooth $Y \subset \bar{Y}$, we apply the long exact sequence $(*)$ above with respect to the Cech hypercovering associated to each proper surjective morphism of the Stein factorization. For the first morphism we use the invariance of rigid cohomology groups of degree 0 for proper surjective morphisms with geometric connected fibers, and for the second morphism we use a certain density of a fiber product which follows from the geometrical unibranchness of varieties in Proposition 4.3.

In section 5 we prove the coincidence of the categories of overconvergent isocrystals and its rigid cohomology groups by inverse images for a separated universal homeomorphism $Y \rightarrow X$ of finite type (Theorem 5.2). Theorem 1.4 follows from these more general comparison results. A key is to show that the constant bi-simplicial scheme gives a resolution both of $X$ and of $Y$ as proper hypercoverings (Proposition 5.1). Then we can apply A. Shiho's proper descent of overconvergent isocrystals [29, Proposition 7.3] and proper cohomological descent of rigid cohomology [32, Theorem 2.3.1]. In the case of convergent isocrystals the proper descent and the above equivalence of categories was studied by A. Ogus [26, Theorem 4.6, Corollary 4.10]. These comparisons are $p$-adic analogues of those in etale cohomology [1, VIII, Théorème 1.1, Corollaire 1.2, Remarques 1.4].

In section 6 we study Frobenius eigenvalues of $H_{\text {rig }}^{1}(X / K)$ for geometrically unibranch $X$ and prove Theorem 1.5 in Theorem 6.3.

In section 7 we give an example such that the natural homomorphism $H_{\text {rig }}^{0}((X, \bar{X}) / K, M) \rightarrow H_{\text {rig }}^{0}\left((U, \bar{X}) / K, j_{U}^{\dagger} M\right)$ is not surjective in the case where $X$ is not geometrically unibranch. Hence, for the full faithfulness of the restriction functor $j_{U}^{\dagger}$, some conditions on $X$ are needed in general. We also give an example such that the restriction functor $j_{U}^{\dagger}$ is not essentially surjective in the case where $X$ is normal. 


\section{Preparations}

Let $j_{X}: X \rightarrow \bar{X}$ be an open immersion of separated schemes locally of finite type over Spec $k, Z$ a closed subscheme of $X, U=X \backslash Z$ with a canonical open immersion $j_{U}: U \rightarrow \bar{X}$, and $M$ an overconvergent isocrystal on $(X, \bar{X}) / K$. Let $L$ be an extension of $K$ as complete discrete valuation fields and $l$ the residue field of $L$. For a commutative diagram

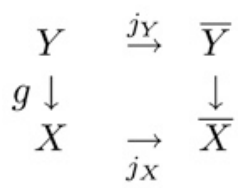

over Spec $k$ such that $j_{Y}$ is an open immersion of separated schemes of locally of finite type over Spec $l$, we denote by $g^{*} M$ the inverse image of $M$ as an overconvergent isocrystal on $(Y, \bar{Y}) / L$.

\section{1 - Fundamental tools in rigid cohomology.}

We recall several fundamental tools, which we will use later, in rigid cohomology.

We recall the definition of the rigid cohomology $H_{\text {rig } Z}^{q}((X, \bar{X}) / K, M)$ on $(X, \bar{X}) / K$ with supports in $Z$ for an overconvergent isocrystal $M$ on $(X, \bar{X}) / K$, which is defined by P. Berthelot [5, Section 2]. In the case where $\bar{X}$ is embedded as a closed subscheme into a separated formal scheme $\mathcal{P}$ locally of finite type over $\operatorname{Spf} \mathcal{V}$ which is smooth around $X$, it is defined by the hypercohomology

$$
H_{\text {rig } Z}^{r}((X, \bar{X}) / K, M):=\mathbb{R}^{r} \Gamma(] \bar{X}\left[\left[_{\mathcal{P}}, \Gamma_{Z}^{\dagger}\left(M \otimes_{j_{X}^{\dagger} \mathcal{O}_{\bar{X}_{I_{\mathcal{P}}}}} j_{X}^{\dagger} \Omega_{]_{\bar{X}}\left[_{\mathcal{P}} / K\right.}^{\bullet}\right)\right) .\right.
$$

Here $j_{X}^{\dagger}$ is an exact functor of overconvergent sections along $\bar{X} \backslash X$ and $\underline{\Gamma}_{Z}^{\dagger}$ is an exact functor of overconvergent sections with supports in $Z$ defined by the kernel of the epimorphism $j_{X}^{\dagger} \rightarrow j_{U}^{\dagger}$ on the category of sheaves of Abelian groups on the tube $] \bar{X}{ }_{\mathcal{P}}$ of $\bar{X}$ in Raynaud's generic fiber $\mathcal{P}_{K}$ associated to $\mathcal{P}$. By definition we have $] \bar{X}\left[_{\mathcal{P}}=\right] \bar{X}_{\text {red }}[\mathcal{P}$ and the identity

$$
H_{\text {rig } Z_{\text {red }}}^{r}\left(\left(X_{\text {red }}, \bar{X}_{\text {red }}\right) / K, M\right)=H_{\text {rig } Z}^{r}((X, \bar{X}) / K, M) .
$$

REMARK 2.1. (1) When one begins with the pair $(X, \bar{X})$, one can find, locally on $\bar{X}$, a closed embedding of $\bar{X}$ into a formal scheme $\mathcal{P}$ over $\operatorname{Spf} \mathcal{V}$ as 
above, and can define overconvergent isocrystals and rigid cohomology on $(X, \bar{X}) / K$ by the simplicial construction using an open covering of $\bar{X}$ in general [7, 1.5 Remarque 4] [13, Section 10.5, 10.6]. All the definitions do not depend on any choices.

(2) When one considers the "full" rigid cohomology, i.e., overconvergent along "full boundaries" (namely " $\bar{X}$ " is proper over Spec $k$ ), one can find, locally on $X$, an open immersion $j_{X}: X \rightarrow \bar{X}$ for a proper scheme $\bar{X}$ over Spec $k$ with an embedding into a formal scheme $\mathcal{P}$ over $\operatorname{Spf} \mathcal{V}$ which is smooth around $X$. Then one can define an overconvergent isocrystal and rigid cohomology on $X / K$ by the simplicial construction using an open covering of $X$ as in the previous remarks. Note that, for a separated scheme $X$ of finite type over Spec $k$, there always exists a completion $\bar{X}$ by Nagata's compactification [22] [23]. In the full boundaries case we use the notation $H_{\text {rig } Z}^{r}(X / K, M)$ instead of $H_{\operatorname{rig} Z}^{r}((X, \bar{X}) / K, M)$.

In this note we always begin with a pair $(X, \bar{X})$, however, the results also hold in the case of overconvergence along full boundaries.

Proposition 2.2 (Finite scalar extensions) [7, Proposition 1.8]. For a finite extension $L$ of $K$, let $l$ (resp. $f: X_{l} \rightarrow X$ ) denote the residue field of $L$ (resp. the extension of base fields). Then, for any $r$, we have a canonical isomorphism

$$
H_{\text {rig } Z_{l}}^{r}\left(\left(X_{l}, \bar{X}_{l}\right) / L, f^{*} M\right) \cong H_{\text {rig } Z}^{r}((X, \bar{X}) / K, M) \otimes_{K} L .
$$

REMARK 2.3. One can find base change theorems of rigid cohomology for an arbitrary scalar extension $L$ of $K$ in [33, Theorem 8.1.1] and [4, Corollary 5.5.2].

Proposition 2.4 [7, Proposition 2.5]

(1) If $Z$ is a disjoint sum of closed subschemes $Z_{1}$ and $Z_{2}$ of $X$, then $H_{\operatorname{rig} Z}^{r}((X, \bar{X}) / K, M) \cong H_{\operatorname{rig} Z_{1}}^{r}((X, \bar{X}) / K, M) \oplus H_{\operatorname{rig} Z_{2}}^{r}((X, \bar{X}) / K, M)$ for any $r$.

(2) (Excision sequence) If $Z_{1}$ is a closed subscheme of $Z$ over Spec $k$, then there is a canonical long exact sequence

$$
\begin{aligned}
& \cdots \rightarrow H_{\text {rig } Z_{1}}^{r}((X, \bar{X}) / K, M) \rightarrow H_{\text {rig } Z}^{r}((X, \bar{X}) / K, M) \rightarrow H_{\text {rig } Z \backslash Z_{1}}^{r}\left(\left(X \backslash Z_{1}, \bar{X}\right) / K, j_{X \backslash Z_{1}}^{\dagger} M\right) \\
& \rightarrow H_{\text {rig } Z_{1}}^{r+1}((X, \bar{X}) / K, M) \rightarrow \\
&
\end{aligned}
$$

We recall some spectral sequences which we will use later (see [33, Sections 2, 5, 7]). 
Proposition 2.5 (Čech spectral sequence for open coverings)

(1) Let $\left\{\bar{Y}_{\lambda}\right\}_{\lambda \in 1}$ be an open covering of $\bar{X}$ over Spec $k$ indexed by a totally order set $\Lambda$, put $\bar{Y}_{\lambda_{0} \lambda_{1} \cdots \lambda_{q}}=\cap_{i=0}^{q} \bar{Y}_{\lambda_{i}}, Y_{\lambda_{0} \lambda_{1} \cdots \lambda_{q}}=X \cap \bar{Y}_{\lambda_{0} \lambda_{1} \cdots \lambda_{q}}$ with the structure morphism $f_{\lambda_{0} \lambda_{1} \cdots \lambda_{q}}: Y_{\lambda_{0} \lambda_{1} \cdots \lambda_{q}} \rightarrow X$, and $W_{\lambda_{0} \lambda_{1} \cdots \lambda_{q}}=Z \cap \bar{Y}_{\lambda_{0} \lambda_{1} \cdots \lambda_{q}}$. Then there is a spectral sequence

$$
\begin{gathered}
E_{1}^{q r}=\underset{\lambda_{0}<\lambda_{1}<\cdots<\lambda_{q}}{\oplus} H_{\text {rigW }}^{r} \underset{\lambda_{0} \lambda_{1} \cdots \lambda_{q}}{(}\left(\left(Y_{\lambda_{0} \lambda_{1} \cdots \lambda_{q}}, \bar{Y}_{\lambda_{0} \lambda_{1} \cdots \lambda_{q}}\right) / K, f_{\lambda_{0} \lambda_{1} \cdots \lambda_{q}}^{*} M\right) \Rightarrow \\
H_{\text {rig } Z}^{q+r}((X, \bar{X}) / K, M) .
\end{gathered}
$$

(2) Let $\left\{Y_{\lambda}\right\}_{\lambda \in A}$ be an open covering of $X$ over Spec $k$ indexed by a totally order set $\Lambda$, put $Y_{\lambda_{0} \lambda_{1} \cdots \lambda_{q}}=\cap_{i=0}^{q} Y_{\lambda_{i}}$ with the structure morphism $f_{\lambda_{0} \lambda_{1} \cdots \lambda_{q}}: Y_{\lambda_{0} \lambda_{1} \cdots \lambda_{q}} \rightarrow X$, and $W_{\lambda_{0} \lambda_{1} \cdots \lambda_{q}}=Z \cap Y_{\lambda_{0} \lambda_{1} \cdots \lambda_{q}}$. Then there is a spectral sequence

$$
\begin{gathered}
E_{1}^{q r}=\underset{\lambda_{0}<\lambda_{1}<\cdots<\lambda_{q}}{\oplus} H_{\text {rigW } W_{\lambda_{0} \lambda_{1} \cdots \lambda_{q}}}^{r}\left(\left(Y_{\lambda_{0} \lambda_{1} \cdots \lambda_{q}}, \bar{X}\right) / K, f_{\lambda_{0} \lambda_{1} \cdots \lambda_{q}}^{*} M\right) \Rightarrow \\
H_{\text {rig } Z}^{q+r}((X, \bar{X}) / K, M) .
\end{gathered}
$$

Proposition 2.6 (Mayer-Vietoris spectral sequence). Suppose $\bar{X}$ is separated of finite type over Spec $k$, and let $\left\{\bar{Y}_{\lambda}\right\}$ be a finite closed covering of $\bar{X}$ over Spec $k$. Then there is a spectral sequence

$$
\begin{gathered}
E_{1}^{q r}=\underset{\lambda_{0}<\lambda_{1}<\cdots<\lambda_{q}}{\oplus} H_{\text {rigW } W_{\lambda_{0} \lambda_{1} \cdots \lambda_{q}}}^{r}\left(\left(Y_{\lambda_{0} \lambda_{1} \cdots \lambda_{q}}, \bar{Y}_{\lambda_{0} \lambda_{1} \cdots \lambda_{q}}\right) / K, f_{\lambda_{0} \lambda_{1} \cdots \lambda_{q}}^{*} M\right) \Rightarrow \\
H_{\mathrm{rig} Z}^{q+r}((X, \bar{X}) / K, M),
\end{gathered}
$$

where $\bar{Y}_{\lambda_{0} \lambda_{1} \cdots \lambda_{q}}=\cap_{i=0}^{q} \bar{Y}_{\lambda_{i}}, \quad Y_{\lambda_{0} \lambda_{1} \ldots \lambda_{q}}=X \cap \bar{Y}_{\lambda_{0} \lambda_{1} \cdots \lambda_{q}}$ with the structure morphism $f_{\lambda_{0} \lambda_{1} \cdots \lambda_{q}}: Y_{\lambda_{0} \lambda_{1} \cdots \lambda_{q}} \rightarrow X$, and $W_{\lambda_{0} \lambda_{1} \cdots \lambda_{q}}=Z \cap Y_{\lambda_{0} \lambda_{1} \cdots \lambda_{q}}$.

A simplicial scheme $T$. over $S$ is a contravariant functor from the standard simplicial category to the category of schemes over $S$, and denotes it by $T_{\bullet} \rightarrow S$. A simplical scheme $T_{\bullet} \rightarrow S$ is said to be a proper hypercovering if the canonical morphism $T_{0} \rightarrow S$ is proper surjective and if the canonical morphism $T_{q+1} \rightarrow \operatorname{cosk}_{q}\left(T_{\bullet}^{(q)}\right)_{q+1}$ is proper surjective for any $q$. Here $T_{\bullet}^{(q)}$ is the truncation of $T$. until the $q$-th stage ( $q$-truncation) and $\operatorname{cosk}_{q}$ is the right adjoint of the $q$-truncation functor. We often use a spectral sequence with respect to a Čech hypercovering associated to a proper surjective morphism, which is defined as follows. A Čech hypercovering $T_{\bullet} \rightarrow S$ associated to a proper surjective morphism $T \rightarrow S$ consists of its $q$-th stage $T_{q}=T \times_{S} \cdots \times_{S} T$ (the fiber product of $q+1$ copies of $T$ over $S$ ) and standard face and degeneracy morphisms. Indeed, 
$\operatorname{cosk}_{q}\left(T_{\bullet}^{(q)}\right)_{q+1}=T_{q+1}$ in this case. Hence, the associated Cech hypercovering $T_{\bullet} \rightarrow S$ is a proper hypercovering.

THEOREM 2.7 (Spectral sequence for proper hypercoverings) [32, Theorem 4.5.1]. Suppose that $\bar{X}$ is separated of finite type over Spec $k$. Let $\bar{g}_{\bullet}: \bar{Y}_{\bullet} \rightarrow \bar{X}$ be a proper hypercovering over Speck, $g_{\bullet}: Y_{\bullet} \rightarrow X$ (resp. $W_{\bullet} \rightarrow Z$ ) the induced proper hypercovering by the inverse image, and $M_{q}$ the inverse image of $M$ as an overconvergent isocrystal on $\left(Y_{q}, \bar{Y}_{q}\right) / K$ by $g_{q}$. Then there is a spectral sequence of rigid cohomology with supports

$$
E_{1}^{q r}=H_{\mathrm{rig} W_{q}}^{r}\left(\left(Y_{q}, \bar{Y}_{q}\right) / K, M_{q}\right) \Rightarrow H_{\mathrm{rig} Z}^{q+r}((X, \bar{X}) / K, M) .
$$

COROLlary 2.8. With the notation as in Theorem 2.7, there is a long exact sequence

$$
\begin{gathered}
0 \rightarrow H_{\operatorname{rig} Z}^{0}((X, \bar{X}) / K, M) \rightarrow E_{1}^{0,0} \rightarrow \operatorname{ker}\left(d_{1}^{1,0}: E_{1}^{1,0} \rightarrow E_{1}^{2,0}\right) \rightarrow \\
H_{\operatorname{rig} Z}^{1}((X, \bar{X}) / K, M) \rightarrow E_{1}^{0,1} .
\end{gathered}
$$

Note that all the properties of rigid cohomology above are functorial in $M,(X, \bar{X}), Z$ and hypercoverings $\left(Y_{\bullet}, \bar{Y}_{\bullet}\right) \rightarrow(X, \bar{X})$. Moreover, when $M$ is an overconvergent $F$-isocrystal, natural homomorphisms between rigid cohomology groups commute with Frobenius.

\section{2 - Cohomological purity}

In the case where $X$ is smooth over Spec $k$, Theorem 1.1 has been already known as cohomological purity by the Gysin map at least when $\bar{X}$ is proper ([7, Théorème 3.8] [30, Theorem 4.1.1]). In general the author can not find a direct proof in literature.

THEOREM 2.9. Suppose that $X$ is a smooth scheme separated of finite type over Spec $k$ and denote the least codimension of generic points of $Z$ in $X$ by e. Then

$$
H_{\text {rig } Z}^{r}((X, \bar{X}) / K, M)=0
$$

for $r<2 e$.

Proof. Suppose $e \geq 1$. Replacing $k$ by a finite extension, we may assume that $\bar{X}$ is geometrically integral by Propositions 2.2 and 2.6. 
After replacing $k$ by a finite extension of $k$ again, there is an open smooth subscheme of the reduced closed subscheme $Z_{\text {red }}$. Hence we may assume that $(X, Z)$ is a smooth pair such that $Z$ is pure of codimension $e$ by Propositions 2.2 and 2.4. Since $(X, Z)$ is a smooth pair, $Z$ is, locally on $X$, an intersection of all irreducible components of a strict normal crossing divisor of $X$ [16, II, Théorème 4.10]. Hence we may assume that there is a strict normal crossing divisor $D=\cup_{i=1}^{e} D_{i}$ of $X$, where $D_{i}$ is an irreducible component, such that $Z=\cap_{i=1}^{e} D_{i}$ by Proposition 2.5. We denote the Zariski closure of $D_{i}$ and $Z$ in $\bar{X}$ by $\bar{D}_{i}$ and $\bar{Z}$, respectively, and put $l_{i}: D_{1} \cap D_{2} \cap \cdots \cap D_{i} \rightarrow X$ to be the closed immersion.

Applying the Čech spectral sequences with respect to a certain open covering of $\bar{X}$ and a certain open covering of $X$ in Proposition 2.5, we may assume that $\bar{X}$ is affine and $X$ is an open subscheme which is defined by $\bar{g} \neq 0$ in $\bar{X}$ for some $\bar{g} \in \Gamma\left(\bar{X}, \mathcal{O}_{\bar{X}}\right)$.

LEMMA 2.10. With the notation as above, there is an affine formal scheme $\mathcal{X}=\operatorname{Spf} \mathcal{A}$ of finite type over $\operatorname{Spf} \mathcal{V}$ such that $\bar{X}$ is an irreducible component of $(\mathcal{X} \times \operatorname{Spf} \mathcal{V} \text { Spec } k)_{\text {red }}$ and $\mathcal{X}$ is smooth over $\operatorname{Spf} \mathcal{V}$ around $X$.

Proof. Let $B$ be a smooth $\mathcal{V}$-algebra of finite type such that $X \cong \operatorname{Spec} B \otimes_{\mathcal{V}} k$. Such a ring $B$ exists by [15, Théorème 6]. Let $C$ be a polynomial ring of finite variables over $\mathcal{V}$ with a $\mathcal{V}$-algebra surjective homomorphism $C \rightarrow \Gamma\left(\bar{X}, \mathcal{O}_{\bar{X}}\right)$ and a $\mathcal{V}$-algebra homomorphism $C \rightarrow B$ such that the diagram

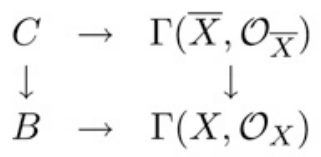

is commutative. Fix a lift $g \in C$ of the element $\bar{g}$. Since $\bar{g}$ is invertible in $\Gamma\left(X, \mathcal{O}_{X}\right)$, we may assume that the image of $g$ in $B$ is invertible. Replacing $C$ by a $\mathcal{V}$-polynomial ring adding variables which goes to generators of $B$ multiplied by suitable powers of $g$, we may assume that the $\mathcal{V}$-algebra homomorphism $C[1 / g] \rightarrow B$ is also surjective. Let $\left(f_{1}, \cdots, f_{r}\right)$ be the kernel of the surjection $C[1 / g] \rightarrow B$ and choose a nonnegative integer $n$ such that all $g^{n} f_{i}$ 's are included in $C$. Define $\mathcal{A}$ by a $p$-adic completion of $C /\left(g^{n} f_{1}, \cdots, g^{n} f_{r}\right)$ and put $\mathcal{X}=\operatorname{Spf} \mathcal{A}$. Since $X$ is open and $\bar{X}$ is closed in $\mathcal{X} \times_{\operatorname{Spf} \mathcal{V}} \operatorname{Spec} k, \bar{X}$ is an irreducible component of $\left(\mathcal{X} \times_{\operatorname{Spf} \mathcal{V}} \operatorname{Spec} k\right)_{\text {red }}$. Moreover, $\mathcal{X}$ is smooth over $\operatorname{Spf} \mathcal{V}$ around $X$ by construction. 
Let us continue the proof of Theorem 2.9. Since the Zariski closure of $X$ in $\left(\mathcal{X} \times_{\text {Spf } \mathcal{V}} \text { Spec } k\right)_{\text {red }}$ is $\bar{X}$, we can replace $\bar{X}$ by $\left(\mathcal{X} \times_{\operatorname{Spf} \mathcal{V}} \text { Spec } k\right)_{\text {red }}$. Applying the Čech spectral sequence with respect to a certain open covering of $X$ in Proposition 2.5, we may assume that each irreducible component $D_{i}$ of $D$ is defined by a single equation $h_{i}=0$ in $X$ for an element $h_{i} \in \mathcal{A}$. Then the desired vanishing follows from the lemma below since $H_{\text {rig } Z}^{r}\left(Z / K, l_{e}^{*} M\right)=H_{\text {rig }}^{r}\left(Z / K, l_{e}^{*} M\right)=0$ for $r<0$.

LEMma 2.11. With the notation above, we have a sequence of isomorphisms

$$
\begin{aligned}
H_{\operatorname{rig} Z}^{r}((X, \bar{X}) / K, M) & \cong H_{\mathrm{rig} Z}^{r-2}\left(\left(D_{1}, \bar{D}_{1}\right) / K, l_{1}^{*} M\right) \cong \cdots \\
& \cong H_{\mathrm{rig} Z}^{r-2 e}\left(\left(D_{1} \cap D_{2} \cap \cdots \cap D_{e}, \bar{D}_{1} \cap \bar{D}_{2} \cap \cdots \cap \bar{D}_{e}\right) / K, l_{e}^{*} M\right) .
\end{aligned}
$$

Proof. Let us put $Y=D_{1}, \bar{Y}=\bar{D}_{1}, l=l_{1}: Y \rightarrow X, h=h_{1}, \mathcal{Y}=$ $\operatorname{Spf} \mathcal{A} /(h)$, and denote Raynaud's generic fiber of $\mathcal{Y}$ by $\mathcal{Y}_{K}$. Then there is a natural closed immersion $\bar{Y} \rightarrow \mathcal{Y}$ such that $\mathcal{Y}$ is smooth around $Y$, and $] \bar{Y}[\mathcal{Y}$ is a closed rigid analytic subspace in $] \bar{X}[\mathcal{X}$ which is defined by $h=0$. We denote the closed immersion by $\left.l_{K}:\right] \bar{Y}[\mathcal{Y} \rightarrow] \bar{X}\left[\mathcal{X}\right.$. Then $l_{K}$ is an affinoid morphism. Moreover, for any open subscheme $T$ in $X$, we have (i) if $V$ is a strict neighborhood of $] T[\mathcal{X}$ in $] \bar{X}[\mathcal{X}$, then $V \cap] \bar{Y}[y$ is a strict neighborhood of $] T \cap Y[\mathcal{y}$ in $] \bar{Y}[\mathcal{y}$, and (ii) if $W$ is a strict neighborhood of $] T \cap Y[\mathcal{y}$ in ] $\bar{Y}[\mathcal{Y}$, then there is a strict neighborhood $V$ of $] T[\mathcal{X}$ in $] \bar{X}[\mathcal{X}$ such that $W \supset V \cap] \overline{\mathcal{Y}}[\mathcal{Y}$ (use standard strict neighborhoods in [6, Examples 1.2.4] to prove (i) and (ii)). It implies that there exists a natural transformation $j_{T}^{\dagger} \rightarrow i_{K *} j_{T \cap Y}^{\dagger}$.

Let $j_{X}^{\dagger} \Omega_{]_{\bar{X}}{ }_{[X} / K}\left(\log \mathcal{Y}_{K}\right)$ be a logarithmic de Rham complex of $] \bar{X}[\mathcal{X}$ over $K$, which is overconvergent along $\bar{X} \backslash X$ with a logarithmic pole along the divisor $h=0$. Since $\mathcal{X}$ is smooth around $X$ and $(X, Y)$ is a smooth pair, there is a strict neighborhood $V$ of $] X[\mathcal{X}$ in $] \bar{X}[\mathcal{X}$ such that $V \cap] \bar{Y}[\mathcal{Y}$ is a smooth divisor of the smooth rigid analytic space $V$. Hence the logarithmic de Rham complex $j^{\dagger} \Omega_{]_{\bar{X}}}^{\bullet} \overline{[X}_{\mathcal{X}}\left(\log \mathcal{Y}_{K}\right)$ is a complex of sheaves of locally free $j_{X}^{\dagger} \mathcal{O}_{] \bar{X}}\left[_{\mathcal{X}}\right.$-modules of finite rank. By taking residues with respect to $d h / h$ we have homomorphisms

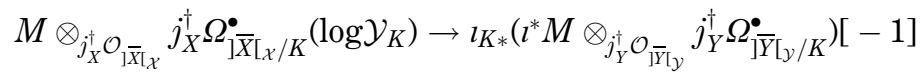

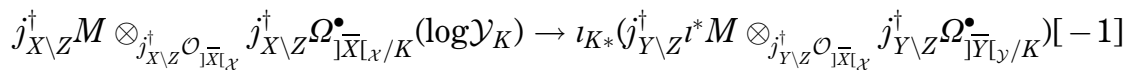

of complexes of $j_{X}^{\dagger} \mathcal{O}_{\bar{X}{ }_{[}{ }_{X}}$-modules and of $j_{X \backslash Z}^{\dagger} \mathcal{O}_{\bar{X}_{\bar{X}}{ }_{X}}$-modules, respectively, where $[a]$ means the $a$-th shift of the complexes, such that the natural 
diagram

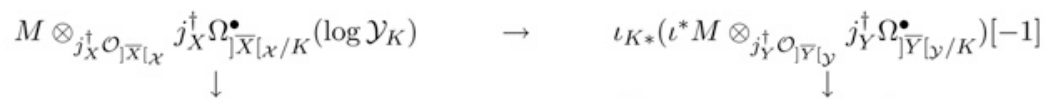

$$
\begin{aligned}
& j_{X \backslash Z}^{\dagger} M \otimes_{j_{X \backslash Z}^{\dagger} \mathcal{O}_{] \bar{X} \backslash \mathcal{X}}} j_{X \backslash Z}^{\dagger} \Omega_{\bar{X}_{[X} / K}^{\bullet}\left(\log \mathcal{Y}_{K}\right) \rightarrow \iota_{K *}\left(j_{Y \backslash Z^{\iota}}^{\dagger} M \otimes_{j_{Y \backslash Z}^{\dagger} \mathcal{O}_{\bar{Y}}[y} j_{Y \backslash Z}^{\dagger} \Omega^{\bullet}{ }_{\bar{Y}[y / K}\right)[-1]
\end{aligned}
$$

is commutative. These diagrams fit into the commutative diagram with short exact rows

$$
\begin{aligned}
& 0 \rightarrow \quad M \otimes_{j_{X}^{\dagger} \mathcal{O}_{|\bar{X}| X}} j_{X}^{\dagger} \Omega_{]_{X}{ }_{[X} / K}^{\bullet} \quad \rightarrow \quad M \otimes_{j_{X}^{\dagger} \mathcal{O}_{\bar{X}_{\lfloor X}}} j_{X}^{\dagger} \Omega_{\bar{X}_{[X} / K}^{\bullet}\left(\log \mathcal{Y}_{K}\right)
\end{aligned}
$$

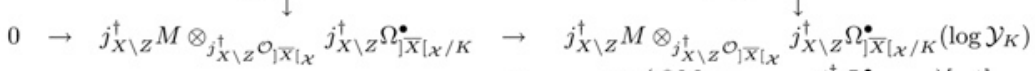

$$
\begin{aligned}
& \rightarrow \quad \iota_{K *}\left(\iota^{*} M \otimes_{j_{Y}^{\dagger} \mathcal{O}_{\bar{Y}}[y} j_{Y}^{\dagger} \Omega_{\bar{Y}_{Y}^{*} \mid y / K}^{\cdot}\right)[-1] \quad \rightarrow 0 \\
& \rightarrow \quad \iota_{K} \cdot\left(j_{Y \backslash Z^{\prime}}^{\dagger} \iota^{*} M \otimes_{j_{Y \backslash Z}^{\dagger} \mathcal{O}_{\mid \bar{Y}} \mid y} j_{Y \backslash Z^{\prime}}^{\dagger} \Omega_{\bar{Y}[y / K}^{\bullet}\right)[-1] \rightarrow 0,
\end{aligned}
$$

where both first arrows in horizontal rows are natural inclusions. The natural inclusions

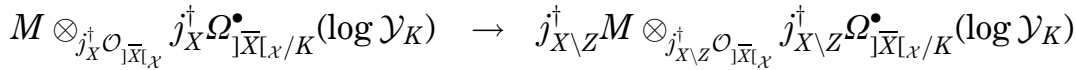

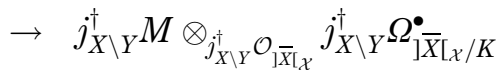

induce isomorphisms

$$
\begin{aligned}
& \operatorname{R} \Gamma(] \bar{X}\left[\mathcal{X}, M \otimes_{j_{X}^{\dagger} \mathcal{O}_{\bar{X}_{\mathcal{X}_{\mathcal{X}}}}} j_{X}^{\dagger} \Omega_{\bar{X}_{\left[_{\mathcal{X}} / K\right.}}^{\bullet}\left(\log \mathcal{Y}_{K}\right)\right) \\
& \cong \mathbb{R} \Gamma(] \bar{X}\left[\mathcal{X}, j_{X \backslash Z}^{\dagger} M \otimes_{j_{X \backslash Z}^{\dagger} \mathcal{O}_{\bar{X}_{\bar{X}_{\mathcal{X}}}}} j_{X \backslash Z}^{\dagger} \Omega_{] \bar{X}_{{ }_{\mathcal{X}} / K} / K}\left(\log \mathcal{Y}_{K}\right)\right)
\end{aligned}
$$

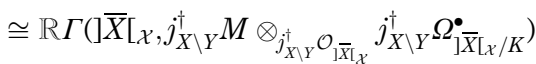

by [10, Theorem 1.1.1, Remarks 1.1.2.4] since all exponents of differential operator along $Y$ are 0 . If $T$ is an open subscheme of $Y$, then $\mathbb{R}_{K *} E=l_{K *} E$ for any coherent sheaf of $j_{T}^{\dagger} \mathcal{O}_{]} \bar{X}_{{ }_{\mathcal{X}}}$-modules [13, Theorem 5.2.1]. Hence we have an isomorphism

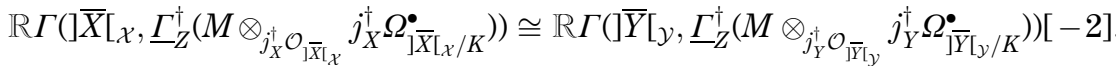

Applying this isomorphism for smooth pairs $\left(X, D_{1}\right),\left(D_{1}, D_{1} \cap D_{2}\right), \cdots$, $\left(D_{1} \cap D_{2} \cap \cdots \cap D_{e-1}, D_{1} \cap D_{2} \cap \cdots \cap D_{e}\right)$ successively, we have obtained the assertion.

REMARK 2.12. (1) The comparison between logarithmic and usual rigid cohomologies were discussed in [2, Theorem A1], [3, Theorem 3.1], [27, Theorem 2.4.9], [30, Theorem 3.5.1] and so on. In each of them the locally 
freeness of $\operatorname{sp}_{*} M$ is assumed, where $\mathrm{sp}: \mathcal{P}_{K} \rightarrow \mathcal{P}$ is the specialization morphism. In [10, Theorem 1.1.1] they do not assume the locally freeness.

(2) The Gysin isomorphism (Lemma 2.11) was studied in more general contexts when $\bar{X}$ is proper in [7, Théorème 3.8] and [30, Theorem 4.1.1].

(3) In this paper we need only the vanishing of $H_{\text {rig } Z}^{0}(X / K, M)$ and $H_{\text {rig } Z}^{1}(X / K, M)$ for a smooth $X$. These follows from Kedlaya's fully faithful theorem of the restriction functor from the category of overconvergent isocrystals on $(X, \bar{X}) / K$ to that of overconvergent isocrystals on $(X \backslash Z, \bar{X}) / K$ [21, Theorem 5.2.1] and the comparison theorem between hom (resp. ext) groups and rigid cohomologies in the next section 2.3.

\section{$2.3-$ Hom and Ext}

We recall the relation between the Hom group (resp. the extension group (Yoneda's Ext group)) of the category of overconvergent isocrystals and the rigid cohomology group $H_{\text {rig }}^{0}$ (resp. $H_{\text {rig }}^{1}$ ).

THEOREM 2.13. Let $M$ and $N$ be overconvergent isocrystals on $(X, \bar{X}) / K$ and $M^{\vee}$ a dual of $M$.

(1) [6, Proposition 2.2.7] There is a natural isomorphism

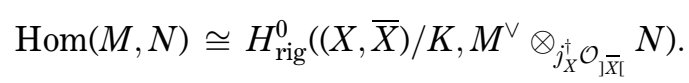

Here Hom means a K-vector space of homomorphisms in the category of overconvergent isocrystals on $(X, \bar{X}) / K$.

(2) [12, Proposition 1.2.2] There is a natural isomorphism

$$
\operatorname{Ext}(M, N) \cong H_{\text {rig }}^{1}\left((X, \bar{X}) / K, M^{\vee} \otimes_{j_{X}^{\dagger} \mathcal{O}_{\bar{X} \mid} \mid} N\right) .
$$

Here Ext means a Yoneda's Ext group in the category of overconvergent isocrystals on $(X, \bar{X}) / K$.

By the definition of overconvergent $F$-isocrystals and [6, Proposition 2.2.7] we have

Corollary 2.14. Suppose that $K$ has a Frobenius $\sigma$. Let $M$ and $N$ be overconvergent $F$-isocrystals on $(X, \bar{X}) / K$ with respect to $\sigma, M^{\vee}$ be a dual of $M$, and

$$
\begin{aligned}
& C(1-F ; M, N)=
\end{aligned}
$$

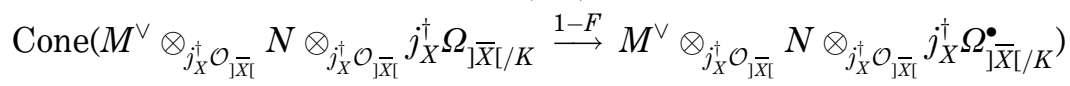


where $F$ is the Frobenius on the de Rham complex induced from that of $M^{\vee} \otimes_{j_{X}^{\dagger} \mathcal{O}_{\bar{X}[}} N$.

(1) There is a natural isomorphism

$$
\operatorname{Hom}(M, N) \cong H^{0}(] \bar{X}[, C(1-F ; M, N)[-1]),
$$

where Hom means a $K^{\sigma}$-space of homomorphisms in the category of overconvergent $F$-isocrystals on $(X, \bar{X}) / K$ and $K^{\sigma}=\{a \in K \mid \sigma(a)=a\}$.

(2) There is a natural isomorphism

$$
\operatorname{Ext}(M, N) \cong H^{1}(] \bar{X}[, C(1-F ; M, N)[-1]),
$$

where Ext means Yoneda's Ext group in the category of overconvergent $F$-isocrystals on $(X, \bar{X}) / K$.

Moreover there is an exact sequence of $K^{\sigma}$-spaces:

$$
\begin{aligned}
& 0 \rightarrow \operatorname{Hom}(M, N) \rightarrow H_{\mathrm{rig}}^{0}\left((X, \bar{X}) / K, M^{\vee} \otimes_{j_{X}^{\dagger} \mathcal{O}_{\bar{J} \mathrm{X}}} N\right) \stackrel{1-F}{\rightarrow} H_{\mathrm{rig}}^{0}\left((X, \bar{X}) / K, M^{\vee} \otimes_{j_{X}^{\dagger} \mathcal{O}_{\bar{X} \bar{X}}} N\right) \\
& \rightarrow \operatorname{Ext}(M, N) \rightarrow H_{\text {rig }}^{1}\left((X, \bar{X}) / K, M^{\vee} \otimes_{j_{X}^{\dagger} \mathcal{O}_{\bar{J} \bar{L}}} N\right) \stackrel{1-F}{\rightarrow} H_{\text {rig }}^{1}\left((X, \bar{X}) / K, M^{\vee} \otimes_{j_{X}^{\dagger} \mathcal{O}_{\bar{X} \mid}} N\right) \text {. }
\end{aligned}
$$

$2.4-$ Alteration

We recall a theorem of the existence of regular alteration by A.J. de Jong [20, Theorem 4.1 and its proof 4.5]. The following statement is what we need in this note.

THEOREM 2.15. Suppose that $X$ is separated of finite type over Spec $k$. Then there is a projective surjective morphism $g: Y \rightarrow X$ such that $Y$ is regular and $g$ is generically finite. Moreover, there exist a purely inseparable finite extension $l$ of $k$ and a projective surjective and generically etale morphism $Y^{\prime} \rightarrow X_{l}$ such that $Y^{\prime}$ is smooth over Spec $l$.

\section{Connectedness.}

3.1 - Connectedness and rigid cohomology groups of degree 0

A rigid analytic space $W$ is connected if there is no admissible covering $\left\{V_{\lambda}\right\}_{\lambda \in \Lambda}$ of $W$ such that $\left(\cup_{\lambda \in \Lambda_{1}} V_{\lambda}\right) \cap\left(\cup_{\lambda \in \Lambda_{2}} V_{\lambda}\right)=\emptyset, \cup_{\lambda \in \Lambda_{1}} V_{\lambda} \neq \emptyset$ and $\cup_{\lambda \in \Lambda_{2}} V_{\lambda} \neq \emptyset$ for a disjoint sum $\Lambda=\Lambda_{2} \amalg \Lambda_{2}[9,9.1 .1]$. An affinoid variety is connected if and only if it is connected with respect to the Zariski topology [9, 9.1.4, Proposition 8]. 
LEMMA 3.1. Let T be a separated scheme offinite type over Spec $k$ with a closed immersion $T \rightarrow \mathcal{P}$ of separated formal schemes of finite type over Spf $\mathcal{V}$ such that $\mathcal{P}$ is smooth around $T$.

(1) Suppose that $k$ is algebraically closed. $] T\left[_{\mathcal{P}}\right.$ is connected if and only if the de Rham cohomology groups $H^{0}(] T\left[_{\mathcal{P}}, \Omega_{] T{ }_{\mathcal{P}} / K}^{\bullet}\right)$ of degree 0 is a $K$ vector space of dimension 1.

(2) If $T$ is connected, then the tube $] T_{{ }_{\mathcal{P}}}$ is a connected rigid analytic space.

The de Rham cohomology group $H^{r}(] T\left[\mathcal{P}, \Omega_{] T[\mathcal{P} / K}^{\bullet}\right)$ in the theorem above is just the rigid cohomology group $H_{\text {rig }}^{r}\left((T, T) / K, \mathcal{O}_{] T[}\right)$ for the unit convergent isocrystal $\mathcal{O}_{] T[}$.

PRoof. (1) Since a constant function is horizontal with respect to connection, the dimension of $\left.H^{0}(] T_{\left[_{\mathcal{P}}\right.}, \Omega_{]_{\left[\left[_{\mathcal{P}} / K\right.\right.}}\right)$ over $K$ is at least the cardinal of connected components of $] T\left[_{\mathcal{P}}\right.$. Hence, if $] T\left[_{\mathcal{P}}\right.$ is not connected, then $\operatorname{dim}_{K} H^{0}(] T\left[\mathcal{P}, \Omega_{] T[\mathcal{P} / K}^{\bullet}\right)>1$.

Suppose that $] T_{\mathcal{P}}$ is connected. Let $t$ be a closed point of $T$. Since $\mathcal{P}$ is smooth over the spectrum of the algebraically closed field $k$, the completion $\widehat{\mathcal{O}_{\mathcal{P}, t}}$ along $t$ is isomorphic to the ring $\mathcal{V}\left[\left[x_{1}, \cdots, x_{n}\right]\right]$ of formal power series in $n$ variables over $\mathcal{V}$ for some $n$. Hence the tube $] t[\mathcal{P}$ is an open unit ball of dimension $n$ over $K$, and $\left.H^{0}(] t_{\left[_{\mathcal{P}}\right.}, \Omega_{]_{t[\mathcal{P}} / K}^{\bullet}\right)=K$. Let $s$ be an element of the kernel of the restriction homomorphism $H^{0}(] T\left[_{\mathcal{P}}, \Omega_{T_{T\left[_{\mathcal{P}} / K\right.}}\right) \rightarrow H^{0}(] t\left[_{\mathcal{P}}, \Omega_{] t\left[_{\mathcal{P}} / K\right.}^{\bullet}\right)$. The homomorphism $\mathcal{O}_{] T\left[_{\mathcal{P}}\right.} \rightarrow \mathcal{O}_{] T\left[_{\mathcal{P}}\right.}$ of convergent isocrystal on $(T, T) / K$ induced by the above section $s$ (Theorem 2.13) has a nontrivial kernel. Since the category of overconvergent isocrystals is Abelian [6, Corollaire 2.2.10] and a convergent isocrystal is locally free by [6, Proposition 2.2.3], the kernel should be $\mathcal{O}_{] T\left[_{\mathcal{P}}\right.}$ by the connectedness of $] T\left[{ }_{\mathcal{P}}\right.$. Hence $s$ is 0 and we have $\operatorname{dim}_{K} H^{0}(] T\left[\mathcal{P}, \Omega_{] T[\mathcal{P} / K}^{\bullet}\right)=1$.

(2) It is sufficient to show the connectedness of $] T\left[_{\mathcal{P}}=\right] T_{\text {red }}[\mathcal{P}$ for each irreducible component of $T$. For any extension $L$ of $K$ as complete discrete valuation fields such that $\mathcal{W}$ is the ring of integers of $L$ and the reside field of $\mathcal{W}$ is $l$, the canonical morphism $T_{l} \rightarrow T$ is surjective and the canonical morphism $] T_{l}\left[\mathcal{P}_{\mathcal{W}} \rightarrow\right] T[\mathcal{P}$ is continuous and surjective. Here $\mathcal{P}_{\mathcal{W}}=\mathcal{P} \times{ }_{\text {Spf } \mathcal{V}} \operatorname{Spf} \mathcal{W}$ as formal schemes. Hence we may assume that $k$ is algebraically closed and $T$ is integral.

First we assume that $T$ is smooth over Spec $k$. We may assume that $T$ is affine. Then there is an affine integral scheme $\widehat{T}$ which is smooth of 
finite type over Spec $\mathcal{V}$ by [15, Théorème 6]. Since the affinoid ring of the $p$-adic completion $\mathcal{T}$ of $\widehat{T}$ is an integral domain, so is the affinoid ring of the affinoid variety $] T T_{\mathcal{T}}=\mathcal{T}_{K}$ and hence $] T T_{\mathcal{T}}$ is connected by the remark just before Lemma 3.1. Consider a natural commutative diagram

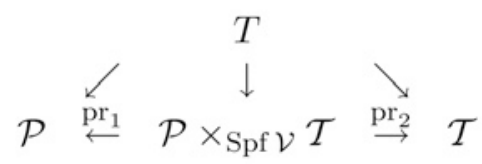

of formal $\mathcal{V}$-schemes such that $T$ is embedded into $\mathcal{P} \times \times_{\operatorname{Spf} \mathcal{V}} \mathcal{T}$ diagonally. Since the rigid cohomology is independent of choices of embeddings, the morphism $\mathrm{pr}_{1}$ and $\mathrm{pr}_{2}$ induce isomorphisms

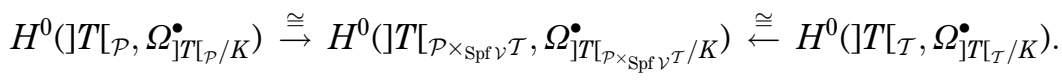

Applying (1), we have $\operatorname{dim}_{K} H^{0}(] T\left[{ }_{\mathcal{P}}, \Omega_{]_{T[\mathcal{P}} / K}\right)=\operatorname{dim}_{K} H^{0}(] T\left[_{\mathcal{T}}, \Omega_{]_{T T_{\mathcal{T}} / K}}\right)=1$. Hence $] T[\mathcal{P}$ is connected again by (1).

Now we prove the assertion in general cases. By applying the existence of regular alteration (Theorem 2.15), there is a projective surjective morphism $X \rightarrow T$ such that $X$ is integral and smooth over $k$. Since $X$ is projective over $T$, there is a smooth projective formal $\mathcal{V}$ scheme $\mathcal{Q}$ over $\mathcal{P}$ with a closed immersion $X \rightarrow \mathcal{Q}$ such that the diagram

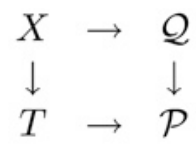

is commutative. Since $\mathcal{Q}$ is smooth over $\operatorname{Spf} \mathcal{V}$ around $X] X,\left[_{\mathcal{Q}}\right.$ is connected. The continuity and surjectivity of the canonical morphism $] X\left[_{\mathcal{Q}} \rightarrow\right] T\left[_{\mathcal{P}}\right.$ imply that $] T[\mathcal{P}$ is connected.

Let $j_{X}: X \rightarrow \bar{X}$ be an open immersion of separated schemes locally of finite type over Spec $k$. Let us denote by $H_{\text {rig }}^{r}((X, \bar{X}) / K)$ the rigid cohomology on $(X, \bar{X}) / K$ for the unit overconvergent isocrystal $j_{X}^{\dagger} \mathcal{O}_{]}{ }_{\bar{X}}$.

Proposition 3.2. Suppose that $k$ is algebraically closed. Then the dimension of $H_{\text {rig }}^{0}((X, \bar{X}) / K)$ as a K-vector space is the cardinal of connected components of $X$. 
Proof. It is sufficient to prove $\operatorname{dim}_{K} H_{\text {rig }}^{0}((X, \bar{X}) / K)=1$ when $X$ is connected. Let $\left\{X_{\lambda}\right\}$ be an open covering of $X$ and suppose $\operatorname{dim}_{K} H_{\text {rig }}^{0}\left(\left(X_{\lambda}, \bar{X}\right) / K\right)=1$ for any $\lambda$. Then $H_{\text {rig }}^{0}\left(\left(X_{\lambda}, \bar{X}\right) / K\right)$ is generated by the identity homomorphism $j_{X_{\lambda}}^{\dagger} \mathcal{O}_{\bar{X}} \rightarrow j_{X_{\lambda}}^{\dagger} \mathcal{O}_{]} \bar{X}[$ by Theorem 2.13. By gluing and Proposition 2.5, $H_{\text {rig }}^{0}\left(\left(X_{\lambda}, \bar{X}\right) / K\right)$ is generated by the identity homomorphism $j_{X_{i}}^{\dagger} \mathcal{O}_{\bar{X}[} \rightarrow j_{X_{\lambda}}^{\dagger} \mathcal{O}_{\bar{X}}$ [ since $X$ is connected. Hence we may assume that $\bar{X}$ is affine and of finite type over Spec $k$. Then one can find a closed immersion $\bar{X} \rightarrow \mathcal{P}$ over Spf $\mathcal{V}$ such that $\mathcal{P}$ is a separated formal scheme of finite type over $\operatorname{Spf} \mathcal{V}$ which is smooth around $X$. Since a constant function is an overconvergent section, the assertion follows from Lemma 3.1.

Corollary 3.3. Let $U$ be an open subscheme of $X$ such that $U \cap Y \neq \emptyset$ for each connected component $Y$ of $X$. Then the restriction map

$$
H_{\text {rig }}^{0}((X, \bar{X}) / K) \rightarrow H_{\text {rig }}^{0}((U, \bar{X}) / K)
$$

is bijective.

ExAmple 3.4. Let $K=Q_{p}(\pi)$ with $\pi^{p-1}=-p, k=\mathbb{F}_{p}, \quad X=\bar{X}=$ Spec $k[x, y] /(x y), X_{1}=\{x=0\} \subset X, X_{2}=\{y=0\} \subset X, U=X \backslash X_{1}$ and embed $X$ in $\mathcal{P}=\widehat{\mathbb{A}}_{\mathcal{V}}^{2}$ with coordinates $x, y$. We define a convergent

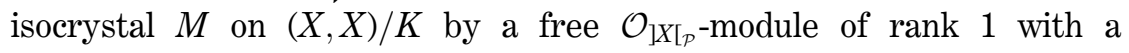
connection

$$
\nabla(1)=-\pi d y
$$

Then, $\left.M\right|_{] X_{1}\left[_{p}\right.}$ is a convergent isocrystal on $\left(X_{1}, X_{1}\right) / K$ which is Dwork's isocrystal corresponding to an additive character, and $\left.M\right|_{X_{X}[p}$ is isomorphic to the unit object $\mathcal{O}_{] X_{2}[p}$ on $\left(X_{2}, X_{2}\right) / K$. Hence $H_{\text {rig }}^{0}((X, X) / K, M)=0$ and $H_{\text {rig }}^{0}\left((U, X) / K, j_{U}^{\dagger} M\right)=K$. It means that the hypothesis of Corollary 3.3 is not enough for the coincidence between $H_{\text {rig }}^{0}((X, \bar{X}) / K, M)$ and $H_{\text {rig }}^{0}\left((U, \bar{X}) / K, j_{U}^{\dagger} M\right)$ in the case with coefficients.

\section{2 - Rank of overconvergent isocrystals.}

If one fixes a closed embedding $\bar{X} \rightarrow \mathcal{P}$ over $\operatorname{Spf} \mathcal{V}$ such that $\mathcal{P}$ is smooth around $X$, then any overconvergent isocrystal on $(X, \bar{X}) / K$ is a locally free $j_{X}^{\dagger} \mathcal{O}_{]_{\bar{X}}{ }_{\mathcal{P}}}$-module of finite rank on the tube $] \bar{X}\left[_{\mathcal{P}}\right.$ [6, Proposition 2.2.3]. It is independent of the choice of embeddings. Moreover, the notion of rank depends only on $X$ : 
Proposition 3.5. Let $X$ be a separated scheme locally of finite type over Spec $k$. Then the rank of a convergent isocrystal on $(X, X) / K$ is stable on each connected component of $X$.

Proof. Since the problem is local on $X$, we may assume that $X$ has a closed embedding into a smooth formal scheme over $\operatorname{Spf} \mathcal{V}$. Since any convergent isocrystal is locally free on the tube, the assertion follows from Lemma 3.1 (2).

\section{3 - Proof of Theorem 1.1 (1).}

We may assume that $X$ is connected and $U$ is nonempty. Take $s \in H_{\text {rig }}^{0}((X, \bar{X}) / K, M)$ such that $j_{U}^{\dagger}(s)=0$, then we will prove $s=0$. Since $s$ determines a homomorphism $\eta: j_{X}^{\dagger} \mathcal{O}_{\bar{X}[} \rightarrow M$ as overconvergent isocrystals on $(X, \bar{X}) / K$ by Theorem 2.13 , the $\operatorname{kernel} \operatorname{ker}(\eta)$ is an overconvergent isocrystal. Indeed, the category of overconvergent isocrystals is Abelian [6, Corollaire 2.2.10]. By the hypothesis of $s$ we have $\left.\operatorname{ker}(\eta)\right|_{] U[} \cong \mathcal{O}_{] U[}$. The connectedness of $X$ implies that the restriction map $\left.\operatorname{ker}(\eta)\right|_{] X[} \rightarrow \mathcal{O}_{] X[}$ is an isomorphism since $\left.\operatorname{ker}(\eta)\right|_{] X[}$ is of rank 1 on $X$ by Proposition 3.5. Hence $\left.s\right|_{] X[}=0$. Since the restriction functor from the category of overconvergent isocrystals on $(X, \bar{X}) / K$ to the category of overconvergent isocrystals on $(X, X) / K$ is faithful [6, Corollaire 2.2.9], we have $s=0$. This completes a proof of Theorem 1.1 (1).

\section{4 - Invariance of rigid cohomology groups of degree 0.}

THEOREM 3.6. Let

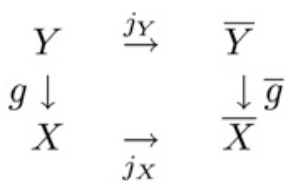

be a commutative diagram of separated schemes locally of finite type over Spec $k$ such that $j_{X}$ and $j_{Y}$ are open immersions, $g$ is proper surjective and $\bar{g}$ is proper on the Zariski closure of $Y$ in $\bar{Y}$. Suppose that the geometric fiber $g^{-1}(x)$ is connected for any geometric point $x$ of $X$ which is above a closed 
point of $X$. Then, for any overconvergent isocrystal $M$ on $(X, \bar{X}) / K$, the natural morphism

$$
g^{*}: H_{\text {rig }}^{0}((X, \bar{X}) / K, M) \rightarrow H_{\text {rig }}^{0}\left((Y, \bar{Y}) / K, g^{*} M\right)
$$

is bijective.

Proof. We may assume that $\bar{g}$ is proper surjective and $\bar{g}^{-1}(X)=Y$. The problem is local on $X$ and $\bar{X}$ by Cech spectral sequence (Proposition 2.5). Hence we may assume that $\bar{X}$ is of finite type over Speck. Let $\bar{g}_{\bullet}: \bar{Y}_{\bullet} \rightarrow \bar{X}$ be a Čech hypercovering associated to the proper surjective morphism $\bar{g}: \bar{Y} \rightarrow \bar{X}$ and put $Y_{\bullet}=\bar{g}_{\bullet}^{-1}(X)$ with the structure morphism $g_{\bullet}: Y_{\bullet} \rightarrow X$.

Applying the spectral sequence to the hypercovering $\left(Y_{\bullet}, \bar{Y}_{\bullet}\right) \rightarrow(X, \bar{X})$ (Theorem 2.7), we have

$$
H_{\text {rig }}^{0}((X, \bar{X}) / K, M)=\operatorname{ker}\left(H_{\text {rig }}^{0}\left(\left(Y_{0}, \bar{Y}_{0}\right) / K, M_{0}\right) \stackrel{d_{1}^{00}}{\rightarrow} H_{\text {rig }}^{0}\left(\left(Y_{1}, \bar{Y}_{1}\right) / K, M_{1}\right)\right)
$$

where $\operatorname{pr}_{i}: \bar{Y}_{1} \rightarrow \bar{Y}_{0}=\bar{Y}$ is the $i$-th projection for $i=0,1$ and $d_{1}^{00}=\mathrm{pr}_{1}^{*}-\mathrm{pr}_{0}^{*}$. In order to obtain a desired natural identification, we need $d_{1}^{00}=0$. Hence, it is sufficient to prove that the morphism

$$
\Delta^{*}: H_{\text {rig }}^{0}\left(\left(Y_{1}, \bar{Y}_{1}\right) / K, M_{1}\right) \rightarrow H_{\text {rig }}^{0}\left((Y, \bar{Y}) / K, g^{*} M\right)
$$

induced by the diagonal $\Delta: \bar{Y} \rightarrow \bar{Y}_{1}$ is injective because $\mathrm{pr}_{0} \circ \Delta=\mathrm{pr}_{1} \circ \Delta$.

Let $s \in H_{\text {rig }}^{0}\left(\left(Y_{1}, \bar{Y}_{1}\right) / K, M_{1}\right)$ be a horizontal section of $M_{1}$ on $] \bar{Y}_{1}[$ such that $\Delta^{*}(s)=0$. Then $s$ determines a homomorphism $\eta: j_{Y_{1}}^{\dagger} \mathcal{O}_{\bar{Y}_{1}[} \rightarrow M_{1}$ as overconvergent isocristals on $\left(Y_{1}, \bar{Y}_{1}\right) / K$ by Theorem 2.13 . Since the category of overconvergent isocrystals is Abelian [6, Corollaire 2.2.10], the kernel $\operatorname{ker}(\eta)$ of $\eta$ is an overconvergent subisocrystal of $j_{Y_{1}}^{\dagger} \mathcal{O}_{\bar{Y}_{1}[}$. In order to prove $s=0$, it is sufficient to prove the rank of the restriction $\left.\operatorname{ker}(\eta)\right|_{{ }_{Y_{1}}[}$ of $\operatorname{ker}(\eta)$ on $\left(Y_{1}, Y_{1}\right) / K$ is always 1 on $Y_{1}$ since the restriction functor from the category of convergent isocrystals on $\left(Y_{1}, \bar{Y}_{1}\right) / K$ to the category of overconvergent isocrystals on $\left(Y_{1}, Y_{1}\right) / K$ is faithful [6, Corollaire 2.2.9]. Since the canonical morphism $\bar{Y}_{1, \bar{k}} \rightarrow \bar{Y}_{1}$, where $\bar{k}$ is an algebraic closure of $k$, is surjective, we have only to prove the assertion in the case where $k$ is algebraically closed.

Let $x$ be a closed point of $X$, and $Y_{x}$ (resp. $Y_{1 x}$ ) the fiber of $g: Y \rightarrow X$ (resp. $g_{1}: Y_{1} \rightarrow X$ ) at $x$. Since the intersection $Y_{1 x} \cap \Delta(Y)$ is nonempty, the rank of $\left.\operatorname{ker}(\eta)\right|_{Y_{1 x} \cap \Delta(Y)[}$ is 1 by the hypothesis of $s$. Since $Y_{x}$ is connected, so is also $Y_{1 x}$ and $\Delta(Y)$ meets any connected component of $Y_{1}$. Hence the rank of $\left.\operatorname{ker}(\eta)\right|_{\}_{1}[}$ is 1 on $Y_{1}$ by Proposition 3.5. This completes a proof. 


\section{Proof of Theorem $1.1(2)$}

\section{1 - The case of proper coverings with geometrically connected fibers}

Let us fix the situation in this and the next sections, 4.1 and 4.2. Let $U \subset X \subset \bar{X}$ be a sequence of open immersions of separated integral schemes of finite type over Spec $k$ with $Z=X \backslash U$ ( $U$ might be empty in section 4.1), and $\bar{g}: \bar{Y} \rightarrow \bar{X}$ a proper and surjective morphism of integral schemes over Spec $k, Y, V$ and $W$ and the inverse images of $X, U$ and $Z$ in $\bar{Y}$, respectively. We denote the structure morphism by $g: Y \rightarrow X$. Let $\bar{g}_{\bullet}: \bar{Y}_{\bullet} \rightarrow \bar{X}$ be a Čech hypercovering induced from the proper surjective morphism $\bar{g}: \bar{Y} \rightarrow \bar{X}$, and $Y_{\bullet}$, (resp. $V_{\bullet}$, resp. $W_{\bullet}$ ) the inverse image of $X$ (resp. $U$, resp. $Z$ ) in $\bar{Y} \bullet$ by $\bar{g} \bullet$ and denote the structure morphism by $g_{\bullet}: Y_{\bullet} \rightarrow X$. Consider a spectral sequence

$$
E_{1}^{q r}=H_{\mathrm{rig} W_{q}}^{r}\left(\left(Y_{q}, \bar{Y}_{q}\right) / K, M_{q}\right) \Rightarrow H_{\mathrm{rig} Z}^{q+r}((X, \bar{X}) / K, M)
$$

with respect to the proper hypercovering $\left(Y_{\bullet}, \bar{Y}_{\bullet}\right) \rightarrow(X, \bar{X})$ in Theorem 2.7.

Proposition 4.1. Suppose that each geometric fiber $g^{-1}(x)$ is connected for any geometric point $x$ of $X$ which is above a closed point of $X$. Then $E_{\infty}^{0,0}=E_{1}^{0,0}$ and $E_{\infty}^{q, 0}=E_{2}^{q, 0}=0$ for any $q \geq 1$. In particular, the natural morphism $g^{*}: H_{\operatorname{rig} Z}^{1}((X, \bar{X}) / K, M) \rightarrow H_{\operatorname{rig} W}^{1}\left((Y, \bar{Y}) / K, g^{*} M\right)$ is injective. Moreover, if furthermore $H_{\mathrm{rig} W}^{1}\left((Y, \bar{Y}) / K, g^{*} M\right)=0$, then we have a vanishing $H_{\operatorname{rig} Z}^{1}((X, \bar{X}) / K, M)=0$.

Proof. What we want is to prove that the edge homomorphism

$$
E_{1}^{q, 0}=H_{\mathrm{rig} W_{q}}^{0}\left(\left(Y_{q}, \bar{Y}_{q}\right) / K, M_{q}\right) \stackrel{d_{1}^{q, 0}}{\longrightarrow} E_{1}^{q+1,0}=H_{\mathrm{rig} W_{q+1}}^{0}\left(\left(Y_{q+1}, \bar{Y}_{q+1}\right) / K, M_{q+1}\right) .
$$

is 0 if $q$ is even and is bijective if $q$ is odd. Note that

$$
d_{1}^{q, 0}=\operatorname{pr}_{1,2, \cdots, q, q+1}^{*}-\operatorname{pr}_{0,2, \cdots, q, q+1}^{*}+\cdots+(-1)^{q+1} \operatorname{pr}_{0,1,2, \cdots, q}^{*}
$$

where $\mathrm{pr}_{?}: \bar{Y}_{q+1} \rightarrow \bar{Y}_{q}$ is the ?-th projection for $?=(1,2, \cdots, q, q+1), \cdots$, $(0,1,2, \cdots, q)$. Since the natural commutative diagram

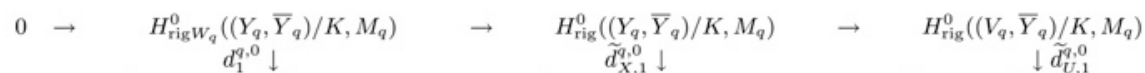

$$
\begin{aligned}
& 0 \rightarrow H_{\mathrm{rig} W_{q+1}}^{0}\left(\left(Y_{q+1}, \bar{Y}_{q+1}\right) / K, M_{q+1}\right) \rightarrow H_{\mathrm{rig}}^{\mathrm{o}}\left(\left(Y_{q+1}, \bar{Y}_{q+1}\right) / K, M_{q+1}\right) \rightarrow H_{\mathrm{rig}}^{0}\left(\left(V_{q+1}, \bar{Y}_{q+1}\right) / K, M_{q+1}\right)
\end{aligned}
$$

arising from the spectral sequences of rigid cohomology groups on $(X, \bar{X})$ and $(U, \bar{X})$ with respect to the proper hypercoverings $\left(Y_{\bullet}, \bar{Y}_{\bullet}\right) \rightarrow(X, \bar{X})$ and 
$\left(V_{\bullet}, \bar{Y}_{\bullet}\right) \rightarrow(U, \bar{X})$ has exact horizontal rows, it is sufficient to prove that $\tilde{d}_{X, 1}^{q, 0}$ and $\widetilde{d}_{U, 1}^{q, 0}$ are 0 -maps if $q$ is even and are bijective if $q$ is odd. If one denotes by $\Delta_{q}: \bar{Y} \rightarrow \bar{Y}_{q}$ (resp. $\Delta_{q+1}: \bar{Y} \rightarrow \bar{Y}_{q+1}$ ) the diagonal morphism, one has $\mathrm{pr}_{\text {? }} \circ \Delta_{q+1}=\Delta_{q}$ for any ?. Since $g: Y \rightarrow X$ is proper with a connected fiber at any geometric point above a closed point of $X$ by our hypothesis, so are $g_{q}: Y_{q} \rightarrow X$ and $g_{q+1}: Y_{q+1} \rightarrow X$. Hence, by applying Theorem 3.6, both $A_{q}^{*}$ and $\Delta_{q+1}^{*}$ are bijective, all $\operatorname{pr}_{?}^{*}: H_{\text {rig }}^{0}\left(\left(Y_{q}, \bar{Y}_{q}\right) / K, M_{q}\right) \rightarrow$ $H_{\text {rig }}^{0}\left(\left(Y_{q+1}, \bar{Y}_{q+1}\right) / K, M_{q+1}\right)$ for ? coincide with each other and are bijective. It implies that $\widetilde{d}_{X, 1}^{q, 0}$ is a 0 -map if $q$ is even and is bijective if $q$ is odd. The same holds for $\widetilde{d}_{U, 1}^{q, 0}$. This completes a proof.

\section{2 - The case of finite coverings}

Keep the notation as in the previous section 4.1 .

Proposition 4.2. Suppose that $U$ is not empty, $\bar{X}$ is geometrically integral, the function field $k(\bar{Y})$ of $\bar{Y}$ is a finite separable extension of the function field $k(\bar{X})$ of $\bar{X}$, and $Y$ is the integral closure of $X$ in $k(\bar{Y})$. If $X$ is geometrically unibranch, then $E_{1}^{q, 0}=H_{\mathrm{rig} W_{q}}^{0}\left(\left(Y_{q}, \bar{Y}_{q}\right) / K, M_{q}\right)=0$ for any $q \geq 0$. In particular, the natural homomorphism

$$
g^{*}: H_{\operatorname{rig} Z}^{1}((X, \bar{X}) / K, M) \rightarrow H_{\operatorname{rig} W}^{1}\left((Y, \bar{Y}) / K, g^{*} M\right)
$$

is injective. Moreover, if furthermore $H_{\mathrm{rig} W}^{1}\left((Y, \bar{Y}) / K, g^{*} M\right)=0$, then we have a vanishing $H_{\operatorname{rig} Z}^{1}((X, \bar{X}) / K, M)=0$.

Proposition 4.2 above follows from Theorem 1.1 (1) and Lemma 4.3 below.

LEMMA 4.3. $V_{q}$ is dense in $Y_{q}$.

LEMMA 4.4. Let $Y_{q}^{\prime}$ be the integral closure of $X$ in $k(\bar{Y})^{\otimes_{k(\bar{X})}}{ }^{q+1}$ for $a$ nonnegative integer $q$, where $k(\bar{Y})^{\otimes_{k(\bar{X})}}{ }^{q+1}$ is a tensor product of $q+1$ copies of $k(\bar{Y})$ over $k(\bar{X})$. Then the natural morphism $h_{q}: Y_{q}^{\prime} \rightarrow Y_{q}$ is surjective.

Proof of Lemma 4.3 Assuming Lemma 4.4. Since $k(\bar{Y})^{\otimes_{k(\bar{X})}}{ }^{q+1}$ is a finite direct product of finite separable extensions of $k(\bar{X}), Y_{q}^{\prime}$ is a finite disjoint union of integral closures of $X$ in some finite separable extensions of $k(\bar{X})$. Hence, if $V_{q}^{\prime}$ is the inverse image of the nonempty $U$ in $Y_{q}^{\prime}$, the topological 
closure $V_{q}^{\prime \text { cl }}$ of $V_{q}^{\prime}$ in $Y_{q}^{\prime}$ is $Y_{q}^{\prime}$ itself. Since $h_{q}\left(V_{q}^{\prime c l}\right)$ is included in the topological closure $h_{q}\left(V_{q}^{\prime}\right)^{\mathrm{cl}}$ of $h_{q}\left(V_{q}^{\prime}\right)$ in $Y_{q}$ and $h_{q}$ is surjective by Lemma 4.4, $V_{q}=h_{q}\left(V^{\prime}\right)$ is dense in $Y_{q}$.

Proof of Lemma 4.4. First we reduce the assertion to the case where $k$ is algebraically closed and $X$ is normal. Let $\bar{k}$ be an algebraic closure of $k$. Consider a natural commutative diagram

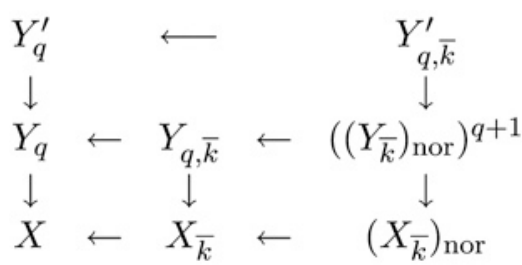

where $Y_{q, \bar{k}}^{\prime}$ is the integral closure of $X_{\bar{k}}$ in the tensor product $\bar{k}(\bar{Y})^{\otimes_{\bar{k} \bar{X} \bar{X}} q+1}$ of $q+1$ copies of $\bar{k}(\bar{Y})$ over $\bar{k}(\bar{X})$ and $\left(\left(Y_{\bar{k}}\right)_{\text {nor }}\right)^{q+1}$ is a fiber product of $q+1$ copies of $\left(Y_{\bar{k}}\right)_{\text {nor }}$ over $\left(X_{\bar{k}}\right)_{\text {nor }}$. Remember that $X$ is geometrically integral and $\bar{k}(\bar{Y})$ is a finite separable extension over $\bar{k}(\bar{X})$ by our hypothesis. Hence $Y_{q, \bar{k}}^{\prime}\left(\right.$ resp. $\left.\bar{k}(\bar{Y})^{\otimes_{\bar{k} \bar{X}(\bar{X}} q+1}\right)$ is a finite disjoint union of integral schemes such that each component is isomorphic to each other (resp. a disjoint sum of finite separable extensions over $\bar{k}\left(\bar{X}_{\bar{k}}\right)$ such that each direct summand is isomorphic to each other).

LEmma 4.5. The morphism $\left(\left(Y_{\bar{k}}\right)_{\text {nor }}\right)^{q+1} \rightarrow Y_{q, \bar{k}}$ in the diagram above is surjective.

Proof. Let $y_{0}, y_{1}, \cdots, y_{q}$ be points in $Y_{\bar{k}}$ such that all $y_{0}, y_{1}, \cdots, y_{q}$ go to $x$ in $X_{\bar{k}}$ by $Y_{\bar{k}} \rightarrow X_{\bar{k}}$. Since $X_{\bar{k}}$ and $Y_{\bar{k}}$ are again geometrically unibranch [19, IV, Propositions 6.15.6, 6.15.7], there exist unique points $y_{0}^{\prime}, \cdots, y_{q}^{\prime} \in\left(Y_{\bar{k}}\right)_{\text {nor }}$ and $x^{\prime} \in\left(X_{\bar{k}}\right)_{\text {nor }}$ such that $y_{0}^{\prime}, \cdots, y_{q}^{\prime}$ and $x^{\prime}$ are above $y_{0}, \cdots, y_{q}$ and $x$, respectively. The uniqueness implies that all $y_{0}^{\prime}, \cdots, y_{q}^{\prime}$ go to $x^{\prime}$ by $\left(Y_{\bar{k}}\right)_{\text {nor }} \rightarrow\left(X_{\bar{k}}\right)_{\text {nor }}$. Hence, we have the surjectivity.

Since $Y_{q, \bar{k}} \rightarrow Y_{q}$ is faithfully flat, it is surjective. In order to prove the surjectivity of $h_{q}: Y_{q}^{\prime} \rightarrow Y_{q}$, we have only to prove the surjectivity of $\left(Y_{q, \bar{k}}\right)^{\prime} \rightarrow\left(\left(Y_{\bar{k}}\right)_{\text {nor }}\right)^{q+1}$. Hence, we may assume that $k$ is algebraically closed and $X$ is normal.

For a scheme $S$ of finite type over Spec $k$, denote by $S_{0}$ the set of closed points of $S$. Then the residue field of $s \in S_{0}$ is $k$ because $k$ is algebraically 
closed. Since a finite morphism is closed, we have $h_{q}\left(\left(Y_{q}^{\prime}\right)_{0}\right) \subset\left(Y_{q}\right)_{0}$. We have only to show $h_{q}\left(\left(Y_{q}^{\prime}\right)_{0}\right)=\left(Y_{q}\right)_{0}$ since the subset of closed points is dense in the case of schemes of finite type over a field. The claim $h_{q}\left(\left(Y_{q}^{\prime}\right)_{0}\right)=\left(Y_{q}\right)_{0}$ and hence Lemma 4.4 follow from the lemma below.

LEMMA 4.6. Let $(A, \mathfrak{m})$ be a normal local domain with a fraction field $E$ such that $k=A / \mathfrak{m}$ is algebraically closed, $F$ a finite separable extension of $E$, and $B$ the integral closure of $A$ in $F$. For a nonnegative integer $q$, let $B^{\otimes_{A} q+1}$ be a tensor product of $q+1$ copies of $B$ over $A$, $F^{\otimes_{E} q+1}$ a tensor product of $q+1$ copies of $F$ over $E, C_{q}$ the integral closure of $A$ in $F^{\otimes_{E} q+1}$, and $h$ : Spec $C_{q} \rightarrow$ Spec $B^{\otimes_{A} q+1}$ the structure morphism induced by the natural A-algebra homomorphism $B^{\otimes_{A} q+1} \rightarrow$ $F^{\otimes_{E}} q+1$. Then $h\left(\left(\operatorname{Spec} C_{q}\right)_{0}\right)=\left(\operatorname{Spec} B^{\otimes_{A} q+1}\right)_{0}$.

Proof. Let $F^{\prime}$ be a Galois closure of the finite separable extension $F / E, B^{\prime}$ the integral closure of $A$ in $F^{\prime}$, and $C_{q}^{\prime}$ the integral closure of $A$ in $\left(F^{\prime}\right)^{\otimes_{E} q+1}$. Consider the natural commutative diagram

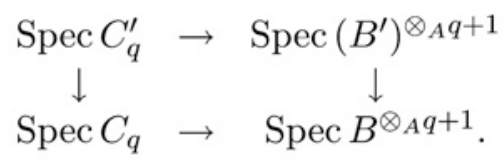

Since the natural morphism $\operatorname{Spec} B^{\prime} \rightarrow \operatorname{Spec} B$ is surjective and since the surjectivity is preserved by any base change, it is sufficient to prove the assertion for the morphism Spec $C_{q}^{\prime} \rightarrow \operatorname{Spec}\left(B^{\prime}\right)^{\otimes_{A} q+1}$. Hence we may assume that the extension $F / E$ is Galois.

Suppose that the extension of $F / E$ is Galois with Galois group $G$. Then $B$ is stable under the action of $G$. Let $\mathfrak{n}_{1}=\mathfrak{n}, \mathfrak{n}_{2}, \cdots, \mathfrak{n}_{r}$ be all maximal ideals of $B$. Then $\mathfrak{m}=\mathfrak{n}_{i} \cap A$ for any $i$ and $(\operatorname{Spec} B)_{0}=\{\sigma(\mathfrak{n}) \mid \sigma \in G\}[24$, Theorem 4.7.7]. Since $B / \mathfrak{m} B$ is a finite $k$-algebra, we have $(B / \mathfrak{m} B)_{\text {red }}=\prod_{i=1}^{r} B / \mathfrak{n}_{i}$. The surjectivity $\left((B / \mathfrak{m} B)_{\text {red }}\right)^{\otimes_{k} q+1} \rightarrow\left((B / \mathfrak{m} B)^{\otimes_{k} q+1}\right)_{\text {red }}$ implies that $B^{\otimes_{A} q+1}$ has exactly $r^{q+1}$ maximal ideals. Moreover, any maximal ideal of $B^{\otimes_{A} q+1}$ is generated by $\sum_{j=0}^{q} l_{j}\left(\sigma_{j}(\mathfrak{n})\right)$ for some $\sigma_{0}, \cdots, \sigma_{q} \in G$, where $l_{j}: B \rightarrow B^{\otimes_{A} q+1}$ is an inclusion into $j$-th component of the tensor product. Say $\mathfrak{n}_{\sigma_{0}, \cdots, \sigma_{q}}$ to be the maximal ideal generated by $\sum_{j=0}^{q} l_{j}\left(\sigma_{j}(\mathfrak{n})\right)$.

Since $F / E$ is Galois, we have $F^{\otimes_{E} q+1}=\prod_{\rho_{1}, \cdots, \rho_{q} \in G} F_{\rho_{1}, \cdots, \rho_{q}}$ where $F_{\rho_{1}, \cdots, \rho_{q}}=F$ and the identification onto the $\left(\rho_{1}, \cdots, \rho_{q}\right)$-component is given 
by $\quad F^{\otimes_{E} q+1} \rightarrow F_{\rho_{1}, \cdots, \rho_{q}}\left(a_{0} \otimes a_{1} \otimes \cdots \otimes a_{q} \mapsto a_{0} \rho_{1}^{-1}\left(a_{1}\right) \cdots \rho_{q}^{-1}\left(a_{q}\right)\right)$. Then $C_{q}=\prod_{\rho_{1}, \cdots, \rho_{q} \in G} B_{\rho_{1}, \cdots, \rho_{q}}$ where $B_{\rho_{1}, \cdots, \rho_{q}}=B$ in $F_{\rho_{1}, \cdots, \rho_{q}}$. Let $\sigma(\mathfrak{n})_{\rho_{1}, \cdots, \rho_{q}}$ be a maximal ideal of $C_{q}$ corresponding to the maximal ideal $\sigma(\mathfrak{n})$ of $\left(\rho_{1}, \cdots, \rho_{q}\right)$ component $B_{\rho_{1}, \cdots, \rho_{q}}=B$, then (Spec $\left.C_{q}\right)_{0}=\left\{\sigma(\mathfrak{q})_{\rho_{1}, \cdots, \rho_{q}} \mid \sigma, \rho_{1}, \cdots, \rho_{q} \in G\right\}$. If we denote the $\left(\rho_{1}, \cdots, \rho_{q}\right)$-component of $h^{\#}: B^{\otimes_{A} q+1} \rightarrow C_{q}$ by $h_{\rho_{1}, \cdots, \rho_{q}}^{\#}: B^{\otimes_{A} q+1} \rightarrow B_{\rho_{1}, \cdots, \rho_{q}}$, then we have

$$
\left(h_{\rho_{1}, \cdots, \rho_{q}}^{\#}\right)^{-1}\left(\sigma(\mathfrak{n})_{\rho_{1}, \cdots, \sigma_{q}}\right)=\mathfrak{n}_{\sigma, \rho_{1} \sigma, \cdots, \rho_{q} \sigma} .
$$

Therefore, $h\left(\left(\operatorname{Spec} C_{q}\right)_{0}\right)=\left(\operatorname{Spec} B^{\otimes_{A} q+1}\right)_{0}$.

\section{3 - First reduction}

Let us start to prove Theorem 1.1 (2). Let us take an open covering $\left\{\bar{Y}_{\lambda}\right\}_{\lambda \in \Lambda}$ of $\bar{X}$ over Spec $k$ by a totally order set $\Lambda$ such that $\bar{Y}_{\lambda}$ is separated of finite type over Spec $k$, and consider a Čech spectral sequence associated to the covering as in Proposition 2.5. Since the hypotheses (i) - (iii) in Theorem 1.1 (2) is local conditions, each $\left(U \cap Y_{\lambda_{0} \ldots \lambda_{q}}, Y_{\lambda_{0} \ldots \lambda_{q}}\right)$ satisfies the hypotheses (i) - (iii) if $Y_{\lambda_{0} \ldots \lambda_{q}} \neq \emptyset$. Hence, Theorem 1.1 (1) implies $E_{1}^{q, 0}=0$ for any $q$. In order to prove $H_{Z}^{1}((X, \bar{X}) / K, M)=0$, it is sufficient to prove $E_{1}^{0,1}=0$. Therefore, we may assume that $\bar{X}$ is separated of finite type over Spec $k$ and $X$ is dense in $\bar{X}$.

Let $\bar{X}=\cup_{\lambda}, \bar{X}_{\lambda}$ be the irreducible decomposition over Spec $k$. If one puts $X_{\lambda}=X \cap \bar{X}_{\lambda}$, then $X_{\lambda} \neq \emptyset$ for all $\eta$ and $X=\cup_{\lambda} X_{\lambda}$ is the irreducible decomposition since $X$ is dense in $\bar{X}$. Replacing $k$ by a finite extension, we may assume that all irreducible components are geometrically irreducible by Proposition 2.2 and [19, IV, Proposition 6.15.7] (the stability of the condition (iii) by extension of base fields). Consider the Mayer-Vietoris spectral sequence in Proposition 2.6 which is associated to the irreducible decomposition. By our hypothesis (ii) Theorem 1.1 (1) implies $E_{1}^{1,0}=0$. In order to prove $H_{\operatorname{rig} Z}^{1}((X, \bar{X}) / K, M)=0$, it is sufficient to prove $E_{1}^{0,1}=0$. Hence we may assume that $\bar{X}$ is geometrically irreducible. Note that the rigid cohomology is determined only by the reduced structures. Therefore, we may assume that $\bar{X}$ is geometrically integral, $U$ is nonempty, and $X$ is geometrically unibranch.

REMARK 4.7. If $\bar{X}$ is separated of finite type over Spec $k$, one can relax the hypothesis (ii) of Theorem 1.1 (2) to the hypothesis: 
(ii)' $U_{\lambda, \mu}$ has a nonempty intersection with each connected component of $X_{\lambda, \mu}$ if $X_{\lambda, \mu} \neq \emptyset$.

Because the "of finite type" hypothesis is required to apply the MayerVietoris spectral sequence (Proposition 2.6).

\section{4 - Applying alteration}

Let us continue the proof of Theorem 1.1 (2). Since we may replace the base field $k$ by a suitable finite extension by Proposition 2.2 and the stability of geometrical unibranchness by extension of base fields [19, IV, Proposition 6.15.7], we may assume that there is a proper surjective and generically etale morphism $\bar{g}: \bar{Y} \rightarrow \bar{X}$ over Spec $k$ such that $\bar{Y}$ is geometrically integral and smooth over Spec $k$ by Theorem 2.15 .

By applying the Stein factorization to $\bar{g}: \bar{Y} \rightarrow \bar{X}$ [18, III, 4.3], the morphism $\bar{g}$ is a composite of two morphisms

$$
\bar{g}: \bar{Y} \rightarrow \bar{Y}^{\prime} \rightarrow \bar{X}
$$

such that

(i) $\bar{Y}^{\prime}$ is the integral closure of $\bar{X}$ in the function field $k(\bar{Y})$ such that $\bar{Y}^{\prime} \rightarrow \bar{X}$ is finite [24, Theorem 4.7.7] and generically etale;

(ii) $\bar{Y} \rightarrow \bar{Y}^{\prime}$ is proper birational.

We denote by $Y^{\prime}, V^{\prime}$ and $W^{\prime}$ (resp. $Y, V$ and $W$ ) the inverse images of $X, U$ and $Z$ in $\bar{Y}^{\prime}$ (resp. $\bar{Y}$ ), respectively, and by $g^{\prime}: Y^{\prime} \rightarrow X$ and $g: Y \rightarrow X$ the structure morphisms. Since $Y$ is smooth and $V$ is dense in $Y$, we have $H_{\text {rig } W}^{1}\left((Y, \bar{Y}) / K, g^{*} M\right)=0$ by Theorem 2.9. Since any geometric fiber of $Y \rightarrow Y^{\prime}$ is connected by Zariski's main theorem [18, III, Théorème 4.3.1], we have $H_{\text {rig } W^{\prime}}^{1}\left(\left(Y^{\prime}, \bar{Y}^{\prime}\right) / K,\left(g^{\prime}\right)^{*} M\right)=0$ by Proposition 4.1. Finally, applying Proposition 4.2, we have the desired vanishing $H_{\operatorname{rig} Z}^{1}((X, \bar{X}) / K, M)=0$.

This completes a proof of Theorem 1.1 (2).

\section{Comparison for universal homeomorphisms}

\section{1 - Resolution by proper hypercoverings}

In this section 5.1 the base scheme $S$ is locally Noetherian. Let $X$ and $Y$ be separated schemes locally of finite type over $S, g: Y \rightarrow X$ a uni- 
versal homeomorphism of finite type, $g_{\bullet}: Y_{\bullet} \rightarrow X$ the Čech hypercovering induced from the proper morphism $g: Y \rightarrow X, C(Y)$. (resp. $D(Y)_{\text {.. }) \text { a }}$ simplicial scheme (resp. a bi-simplicial scheme) such that $C(Y)_{q}=Y$ for any $q$ (resp. $D(Y)_{q, r}=Y$ for any $q$ and $r$ ) and any face map (resp. any degeneracy map) is the identity map $\operatorname{id}_{Y}$. In other words, $C(Y)$. (resp. $D(Y)$..) is the constant simplicial (resp. bi-simplicial) scheme. Since the diagram

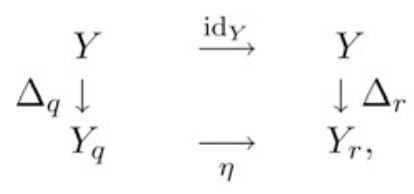

where $\Delta_{q}$ and $\Delta_{r}$ are the diagonal morphisms, is commutative for any face or degeneracy map $\eta$, the first projection

$$
D(Y) \bullet \bullet Y_{\bullet}
$$

is a morphism as a bi-simplicial object over a simplical scheme over $S$, that is,

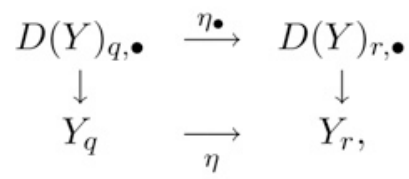

is a morphism of simplicial schemes for any morphism $\eta: Y_{q} \rightarrow Y_{r}$ in the standard simplicial category.

Proposition 5.1. With the notation as above, the diagonal closed immersion $\Delta_{q}: Y \rightarrow Y_{q}$ is surjective. In particular, $D(Y)_{q, \bullet} \rightarrow Y_{q}$ is a Čech hypercovering associated to the proper surjective morphism $Y \rightarrow Y_{q}$ for any $q$.

Proof. By definition of universal homeomorphisms $\Delta_{q}$ is a homeomorphism by induction on $q$. Since $\Delta_{q}$ is a closed immersion, the fiber product of finite copies of $Y$ over $Y_{q}$ is isomophic to $Y$ by the diagonal morphism. Hence $D(Y)_{q \bullet} \rightarrow Y_{q}$ is a Čech hypercovering associated to $\Delta_{q}: Y \rightarrow Y_{q}$.

By Proposition 5.1 we have a commutative diagram 
$(\star)$

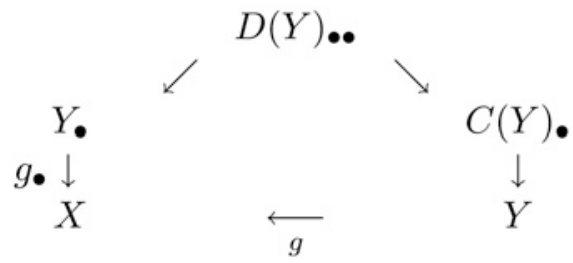

of diagram of schemes over $S$, where $D(Y)_{\bullet \bullet} \rightarrow Y_{\bullet}\left(\right.$ resp. $\left.D(Y)_{\bullet \bullet} \rightarrow C(Y)_{\bullet}\right)$ is the first (resp. second) projection, such that all of $D(Y)_{q, \bullet} \rightarrow$ $Y_{q}, D(Y)_{\bullet}, r \rightarrow C(Y)_{r}, C(Y)_{\bullet} \rightarrow Y$, and $Y \bullet \rightarrow X$ are proper hypercoverings.

\section{2 - Comparison theorem}

Now we return to the case of characteristic $p>0$. Let $S$ be a separated scheme locally of finite type over Spec $k$, and $\subseteq$ a separated formal scheme locally of finite type over $\operatorname{Spf} \mathcal{V}$. Let

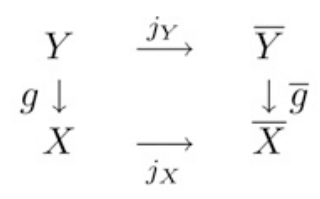

be a commutative diagram of separated scheme locally of finite type over $S$ with structure morphisms $f: X \rightarrow S$ and $h: Y \rightarrow S$ such that both $j_{X}$ and $j_{Y}$ are open immersions, $g$ is of finite type, and $\bar{g}$ is proper on the Zariski closure of $Y$ in $\bar{Y}$. When one is given $X, \bar{X}$ and $Y$, one can find $\bar{Y}$ if $\bar{X}$ is quasicompact by the compactification in [14, Theorem 4.1]. The definition of overconvergent isocrystals on $(X, \bar{X}) / \widetilde{S}_{K}$ and its relative rigid cohomology is found in [13, section 10].

THEOREm 5.2. Suppose that $g: Y \rightarrow X$ is a universal homeomorphism.

(1) The inverse image functor $g^{*}$ from the category of overconvergent isocrystals on $(X, \bar{X}) / \Im_{K}$ to the category of overconvergent isocrystals on $(Y, \bar{Y}) / \mathbb{S}_{K}$ gives an equivalence of categories. When $\mathcal{V}$ and $\subseteq$ admit compatible lifts $\sigma$ and $\sigma_{\Im}$ of Frobenius, then the similar equivalence holds for overconvergent $F$-isocrystals with respect to $\sigma_{\Xi}$.

(2) The natural homomorphism

$$
g^{*}: \mathbb{R}^{r} f_{\text {rig } \varsigma_{K^{*}}} M \rightarrow \mathbb{R}^{r} h_{\text {rig } \Im_{K} *} g^{*} M
$$


between relative rigid cohomology sheaves is an isomorphism for any overconvergent isocrystal $M$ on $(X, \bar{X}) / \widetilde{\Xi}_{K}$. In particular, if $S=$ Spec $k$ and $\subseteq=\operatorname{Spf} \mathcal{V}$, the natural homomorphism

$$
g^{*}: H_{\text {rig } Z}^{r}((X, \bar{X}) / K, M) \rightarrow H_{\text {rig } W}^{r}\left((Y, \bar{Y}) / K, g^{*} M\right)
$$

is an isomorphism for any closed subscheme $Z$ of $X$ with $W=g^{-1}(Z)$.

In order to prove (1) of the theorem above we review Shiho's proper descent for overconvergent isocrystals [29, Proposition 7.3]. An overconvergent isocrystal $M_{\bullet}$ on $\left(Y_{\bullet}, \bar{Y}_{\bullet}\right) / \widetilde{\Im}_{K}$ is a collection of an overconvergent isocrystal $M_{q}$ on $\left(Y_{q}, \bar{Y}_{q}\right) / \widetilde{\Xi}_{K}$ for all $q$ such that there is an isomorphism $u(\eta): \eta^{*} M_{r} \stackrel{\cong}{\longrightarrow} M_{q}$ for any morphism $\eta: X_{q} \rightarrow X_{r}$ in the standard simplicial category which satisfies the conditions (i) $u(\xi \eta)=$ $u(\eta) \eta^{*}(u(\xi))$ and (ii) $u\left(\mathrm{id}_{X_{q}}\right)=\mathrm{id}_{M_{q}}$. An overconvergent isocrystal on $M_{\text {.• }}$ (resp. $\left.M_{\bullet}\right)$ on $\left(D(Y)_{. .}, D(\bar{Y})_{. \bullet}\right) / \widetilde{\Xi}_{K}\left(\operatorname{resp} .\left(C(Y)_{\bullet}, C(\bar{Y})_{\bullet}\right) / \widetilde{\Xi}_{K}\right)$ is also defined similarly. Because the descent data [28, Section 5] is involved in the conditions (i) and (ii). Hence, Shiho's proper descent is as follows by Proposition 5.1.

Theorem 5.3 [29, Proposition 7.3]. Both inverse image functors

$$
\begin{aligned}
\left(\begin{array}{c}
\text { overconvergent isocrystals } \\
\text { on }(X, \bar{X}) / \Im_{K}
\end{array}\right) & \rightarrow\left(\begin{array}{c}
\text { overconvergent isocrystals } \\
\text { on }\left(Y_{\bullet}, \bar{Y} \bullet / \widetilde{\Xi}_{K}\right.
\end{array}\right) \\
& \rightarrow\left(\begin{array}{c}
\text { overconvergent isocrystals } \\
\operatorname{on}\left(D(Y)_{. .}, D(\bar{Y})_{\text {.. }}\right) / \widetilde{\Xi}_{K}
\end{array}\right)
\end{aligned}
$$

are equivalences of categories.

Shiho assumed that the hypercovering is a "strongly" Cech proper hypercovering, that is, $\bar{g}^{-1}(X)=Y$ and $\bar{g}$ is proper and surjective. But one can relax the hypothesis because the category of overconvergent isocrystals does not depend on the choice of compactifications. More precisely, if one takes another $\bar{X}^{\prime}$ with a commutative diagram

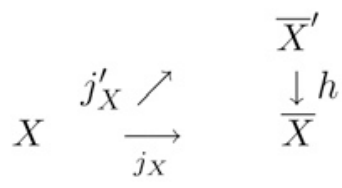

such that $j_{X}^{\prime}$ is an open immersion and $h$ is proper, then the category of overconvergent isocrystals on $(X, \bar{X}) / \widetilde{S}_{K}$ and that on $\left(X, \bar{X}^{\prime}\right) / \widetilde{S}_{K}$ are 
equivalent (resp. the natural homomorphism $\mathbb{R}^{r} f_{\text {rig } \Im_{K^{*}}} M \rightarrow \mathbb{R}^{r} f_{\text {rig } \Im_{K^{*}}}^{\prime} M^{\prime}$ of relative rigid cohomology sheaves is an isomorphism, where $M^{\prime}$ is the inverse image of $M$ and the direct image of $f^{\prime}$ means the one for $\left(X, \bar{X}^{\prime}\right)$ over $\widetilde{\Im}_{K}$ ) [6, Théorème 2.3.5] [13, Proposition 10.2.11] (resp. [13, Proposition 10.5.3]).

Now we prove Theorem 5.2. Since the equivaleces of categories of

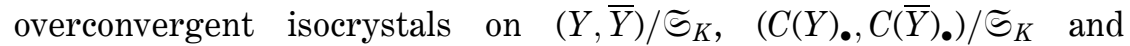
$\left(D(Y)_{. \bullet}, D(\bar{Y})_{. \bullet}\right) / \Im_{K}$ is clear. Hence, the assertion (1) follows Theorem 5.3 and the commutativity of the diagram $(\star)$ in section 5.1 . The assertion (2) follows from the proper cohomological descent of rigid cohomology [32, Theorem 2.1.3] and the above remark of the independence of the choices of compactifications. For the rigid cohomology with supports, the excision sequence in Proposition 2.4 is also applied.

REMARK 5.4. One can prove the comparison as above by this method (see [16, IX, Théorème 4.10]) in any reasonable cohomology theory furnishing the expected properties including proper descent of sheaves and proper cohomological descent, for example, Betti cohomology, etale cohomology and so on.

\section{Application to Frobenius eigenvalues}

Let $K$ be a finite extension of the field $Q_{p}$ of $p$-adic numbers with residue field $k$ of $p^{s}$ elements. Suppose that $K$ admits a Frobenius endomorphism $\sigma$, i.e., a continuous lift of the absolute Frobenius endomorphism $a \mapsto a^{p}$ on $k$ such that the $s$-th iteration $\sigma^{s}$ of $\sigma$ is the identity on $K$. We fix an isomorphism between an algebraic closure $\bar{K}$ of $K$ and the field $\mathbb{C}$ of complex numbers. For integers $w_{1} \leq w_{2}$, [w $\left.w_{1}, w_{2}\right]$ denotes the set of integers $w$ with $w_{1} \leq w \leq w_{2}$.

Let $V$ be a $K$-vector space of finite dimension with a $K$-linear bijective endomorphism $\Phi: V \rightarrow V$. We say that $(V, \Phi)$ is mixed of weights in $\left[w_{1}, w_{2}\right]$ for some integers $w_{1} \leq w_{2}$ if, for any eigenvalue $\alpha$ of $\Phi$, (i) $\alpha$ is an algebraic number and (ii) there is an integer $w \in\left[w_{1}, w_{2}\right]$ such that the complex absolute values of all conjugations of $\alpha$ over $\mathrm{Q}$ are $p^{s w / 2}$. We say that $(V, \Phi)$ is pure of weight $w$ if $(V, \Phi)$ is mixed of weights in $[w, w]$.

Let $X$ be a separated scheme of finite type over Spec $k, H_{\text {rig }}^{r}(X / K)$ the $r$ th rigid cohomology group for the unit overconvergent isocrystal $j_{X}^{\dagger} \mathcal{O}_{\bar{X}}[$ where $\bar{X}$ is a compactification of $X$ over Spec $k$. Let $F: H_{\text {rig }}^{r}(X / K) \rightarrow$ 
$H_{\text {rig }}^{r}(X / K)$ be the Frobenius endomorphism which is induced by the absolute Frobenius on $X$. Then $H_{\text {rig }}^{r}(X / K)$ is a finite dimensional $K$-vector space and $F$ is a $\sigma$-linear bijective endomorphism [32, Theorem 5.1.1]. The $s$-th iteration $F^{s}$ of $F$ is $K$-linear.

Theorem 6.1 ([11] Theorem 1.2 for proper cases and Section 2 for open cases). Suppose that $X$ is smooth and purely of dimension d over Spec $k$. Then $\left(H_{\mathrm{rig}}^{r}(X / K), F^{s}\right)$ is mixed of weights in $[r, \min \{2 r, 2 d\}]$. Moreover, if $X$ is proper over Spec $k$, then $\left(H_{\text {rig }}^{r}(X / K), F^{s}\right)$ is pure of weight $r$.

For a general $X$ we do not know even the vanishing of usual rigid cohomology group $H_{\text {rig }}^{r}(X / K)$ for $r>2 \operatorname{dim} X$. We only know that $H_{\text {rig }}^{r}(X / K)=0$ for sufficient large $r$ [33, Proposition 6.4.1]. Note that the weights for rigid cohomology with compact supports was already studied in [25, Theorem 15.11].

THeorem 6.2 [32, Corollary 5.2.3 for proper cases]. If $X$ is of dimension $\leq d$, then $\left(H_{\mathrm{rig}}^{r}(X / K), F^{s}\right)$ is mixed of weights in $[0, \min \{2 r, 2 d\}]$. Moreover, if $X$ is proper over Spec $k$, then $\left(H_{\text {rig }}^{r}(X / K), F^{s}\right)$ is mixed of weights in $[0, r]$.

Proof. One can construct a proper hypercovering $Y_{\bullet} \rightarrow X$ (resp. when $X$ is proper over Spec $k$ ) such that each $Y_{q}$ is smooth (resp. proper smooth) over Spec $k$ of dimension $\leq d$ and one has a spectral sequence

$$
E_{1}^{q r}=H_{\text {rig }}^{r}\left(Y_{q} / K\right) \Rightarrow H_{\text {rig }}^{q+r}(X / K)
$$

in Theorem 2.7 (see [33, Theorem 7.1.2]). The assertion follows from Theorem 6.1 .

In geometrically unibranch cases the weights of Frobenius in the first rigid cohomology is close to that for smooth cases.

THEOREM 6.3. Suppose that $X$ is geometrically unibranch. Then $\left(H_{\text {rig }}^{1}(X / K), F^{s}\right)$ is mixed of weights in $[1,2]$. Moreover, if $X$ is proper over Spec $k$, then $\left(H_{\text {rig }}^{1}(X / K), F^{s}\right)$ is pure of weight 1 .

In the next lemma the base field $k$ is arbitrary of characteristic $p>0$.

LEMma 6.4. Let $g: Y \rightarrow X$ be a proper surjective morphism of schemes separated of finite type over Spec $k$. Suppose that $X$ is geometrically uni- 
branch. Then the natural homomorphism

$$
g^{*}: H_{\text {rig }}^{1}(X / K) \rightarrow H_{\text {rig }}^{1}(Y / K)
$$

is injective.

We will give an example which tells us the natural homomorphism $g^{*}: H_{\text {rig }}^{1}(X / K) \rightarrow H_{\text {rig }}^{1}(Y / K)$ is not bijective in Example 7.2.

Proof of Lemma 6.4. Note that we may replace the base field $k$ by a finite extension of $k$ by Proposition 2.2. Let $\bar{X}$ be a compactification of $X$ over Spec $k$. Since $X$ is geometrically unibranch, one can deal with the problem on each irreducible component of $X$. Hence, we may assume that $X$ is geometrically integral.

Taking the reduced closed subscheme of an irreducible component of $Y$, we may assume that $Y$ is also integral by Propositions 2.2 and 2.6. By the functoriality of inverse images on rigid cohomology groups we may assume that $Y$ is normal and there exists a compactification $\bar{Y}$ of $Y$ over $\bar{X}$ such that $\bar{Y}$ is also a normal integral scheme by Nagata's compactification [22] [23]. Let

$$
\bar{Y} \rightarrow \bar{Y}^{\prime} \rightarrow \bar{X}
$$

be the Stein factorization of $\bar{Y} \rightarrow \bar{X}$. Then $\bar{Y}^{\prime}$ is normal and is finite over $\bar{X}$ by the normality of $\bar{Y}$, and $\bar{Y} \rightarrow \bar{Y}^{\prime}$ has a connected fiber at each geometric point of $\bar{Y}^{\prime}$. Put $Y^{\prime}$ to be the inverse image of $X$ in $\bar{Y}^{\prime}$. If we consider a spectral sequence

$$
E_{1}^{q r}=H_{\mathrm{rig}}^{r}\left(\widetilde{Y}_{q} / K\right) \Rightarrow H_{\mathrm{rig}}^{q+r}\left(Y^{\prime} / K\right)
$$

with respect to the Čech proper hypercovering $\tilde{Y}_{\bullet} \rightarrow Y^{\prime}$ associated to $Y \rightarrow Y^{\prime}$, then we have $E_{\infty}^{q 0}=E_{2}^{q 0}=0$ for $q \geq 1$ by the proof of Proposition 4.1. Thus $H_{\text {rig }}^{1}\left(Y^{\prime} / K\right)$ is a subspace of $H_{\text {rig }}^{1}(Y / K)$. Hence, we may assume that $Y \rightarrow X$ is generically finite.

Since $\bar{X}$ is geometrically integral, there exists an open dense subscheme of $X$ which is smooth over Spec $k$. By applying the existence of regular alteration in Theorem 2.15, there is a proper surjective and generically finite morphism $Y^{\prime} \rightarrow Y$ such that $Y^{\prime}$ is smooth over Spec $k$ if we replace $k$ by a finite extension again. Hence we may assume that $Y$ is smooth over Spec $k$. Hence there exists an open dense affine subscheme $U$ of $X$ which is smooth over Spec $k$ such that the inverse image $V$ of $U$ in $Y$ is finite and flat. By comparing rigid cohomology with Monsky-Washnitzer cohomology and using a retraction map of Monsky-Washnitzer cohomology with re- 
spect to the finite flat morphism $V \rightarrow U$, the natural homomorphism $H_{\text {rig }}^{1}(U / K) \rightarrow H_{\text {rig }}^{1}(V / K)$ is injective [7, Proposition 3.6].

If we put $Z=X \backslash U$ and $W=Y \backslash V$, then there is a natural commutative diagram

$$
\begin{array}{ccccc}
H_{\text {rig } Z}^{1}(X / K) & \rightarrow & H_{\text {rig }}^{1}(X / K) & \rightarrow & H_{\text {rig }}^{1}(U / K) \\
\downarrow & & \downarrow & & \downarrow \\
H_{\text {rig } W}^{1}(Y / K) & \rightarrow & H_{\text {rig }}^{1}(Y / K) & \rightarrow & H_{\text {rig }}^{1}(V / K)
\end{array}
$$

with exact rows, and $H_{\operatorname{rig} Z}^{1}(X / K)=0$ by Theorem 1.1 (2). Therefore, $g^{*}: H_{\text {rig }}^{1}(X / K) \rightarrow H_{\text {rig }}^{1}(Y / K)$ is injective.

Proof OF TheOREM 6.3. By Theorem 2.15 there is a proper surjective morphism $g: Y \rightarrow X$ such that $Y$ is smooth over Spec $k$ since $k$ is perfect. Hence the assertion follows from Theorem 6.1 and Lemma 6.4.

\section{Examples}

7.1 - An example in non geometrically unibranch case

EXAMPLE 7.1 Let $p$ be an odd prime number, $K=\mathrm{Q}_{p}, k=\mathbb{F}_{p}$, and $\sigma=\mathrm{id}_{\mathrm{Q}_{p}}$ the Frobenius endomorphism. Let $\bar{X}=X$ be a plane curve defined by $x_{0} x_{2}^{2}=x_{1}^{3}+x_{1}^{2} x_{0}$ in the projective plane $\mathbb{P}_{k}^{2}$ over Spec $k$, where $x_{0}, x_{1}, x_{2}$ are homogeneous coordinates, and $Z=[1: 0: 0] \in X$. Then $U=X \backslash Z \cong \mathrm{G}_{\mathrm{m}, k}$. Let $Y=\bar{Y}$ be the normalization of $X$. Then $Y$ is isomorphic to the projective line and the inverse image $W$ of $Z$ in $Y$ consists of two distinct $k$-rational points $s, t$. Hence $X$ is not unibranch.

Using the Poincaré duality with respect to $U$ [8, Théorème 3.4] and the excision sequence of rigid cohomology with compact support

$$
0 \rightarrow H_{\mathrm{rig}, c}^{0}(U / K) \rightarrow H_{\text {rig }}^{0}(X / K) \rightarrow H_{\mathrm{rig}}^{0}(Z / K) \rightarrow H_{\mathrm{rig}, c}^{1}(U / K) \rightarrow \cdots
$$

(see [7, Remark 3.9]), we have

$$
H_{\text {rig }}^{r}(X / K)=\left\{\begin{array}{cl}
K(0) & \text { if } r=0,1 \\
K(-1) & \text { if } r=2, \\
0 & \text { otherwise. }
\end{array}\right.
$$

Here $K(a)$ means the $a$-th Tate twist of $F$-isocrystal on Spec $k / K$, i.e., Frobenius acts by multiplying by $p^{-a}$. By applying the excision sequence of 
rigid cohomology and by using the argument of weights, we have

$$
H_{\text {rig } Z}^{r}(X / K)=\left\{\begin{array}{cl}
K(0) & \text { if } r=1, \\
K(-1) \oplus K(-1) & \text { if } r=2, \\
0 & \text { otherwise. }
\end{array}\right.
$$

Let $M$ be an extension of $\mathcal{O}_{] X[}$ by $\mathcal{O}_{] X[}$ which corresponds to a nontrivial element of the image of $H_{\mathrm{rig} Z}^{1}(X / K) \rightarrow H_{\mathrm{rig}}^{1}(X / K)$. Then $M$ is a convergent isocrystal on $X / K$, which is not split on $X / K$, by Theorem 2.13. Since $j_{U}^{\dagger} M$ is split on $(U, X) / K$, the natural restriction map

$$
H_{\text {rig }}^{0}(X / K, M) \rightarrow H_{\text {rig }}^{0}\left(U / K, j_{U}^{\dagger} M\right)
$$

is not surjective.

The reason why the proof of Theorem 1.1 (2) does not work in the case above is as follows. By taking an irreducible decomposition, there is a surjective closed immersion $\widetilde{Y}_{1}=Y \coprod(s, t) \coprod(t, s) \rightarrow Y_{1}=Y \times_{X} Y$, where $Y$ is embedded diagonally into $Y_{1}$. Hence, $V_{1}=V \times{ }_{U} V \cong U$ is not dense in $Y_{1}$. Moreover, there is a proper hypercovering $\widetilde{Y}_{\bullet} \rightarrow X$ which is defined by

$$
\widetilde{Y}_{q}=Y \coprod \coprod_{\substack{a \in\{s, t\}^{q+1} \\ a \neq(s, \cdots, s)(t, \cdots, t)}} a \text {. }
$$

Hence, we have $H_{\operatorname{rig} Z}^{1}(X / K)=E_{\infty}^{1,0}=E_{2}^{1,0}=K(0)$. One can also find an $F$ isocrystal $M$ on $X / K$ such that $M$ is not split on $X / K$ and $j_{U}^{\dagger} M$ is split on $(U, X) / K$ as an overconvergent $F$-isocrystal by Corollary 2.14 .

\section{2 - An example of a normal case of dimension 2}

We give an example such that the restriction functor $j_{U}^{\dagger}$ from the category of overconvergent isocrystals on $X / K$ to the category of overconvergent isocrystals on $U / K$ is not essentially surjective.

EXAMPLE 7.2 Let $p$ be a prime number with $p \neq 3, K=Q_{p}, k=\mathbb{F}_{p}$, and $\sigma=\mathrm{id}_{\mathrm{Q}_{p}}$ the Frobenius endomorphism. Let $\bar{X}=X$ be a surface defined by $x_{3}^{3}=x_{1} x_{2}\left(x_{1}-x_{2}\right)$ in the projective space $\mathbb{P}_{k}^{3}$ over Spec $k$ of dimension 3 , where $x_{0}, x_{1}, x_{2}, x_{3}$ are homogeneous coordinates, $Z=[1: 0: 0: 0] \in X$, and $U=X \backslash Z$. Then $X$ is normal since $X$ is a complete intersection with the only singular point $Z$ of codimension 2 by Serre's criterion of normality [19, IV, Théorème 5.8.6]. 
Let us take an open covering $U=U_{1} \cup U_{2}$, where $U_{i}$ is defined by $x_{i} \neq 0$. Then $U_{1}$ (resp. $U_{2}$ ) is a product of $A_{k}^{1}$ and $E \backslash\{P\}$ (resp. $E \backslash\{Q\}$ ), where $A_{k}^{1}$ is the affine line, $E$ is an elliptic curve defined by the homogeneous equation $z^{3}=x y(x-y)$, and $P_{1}$ (resp. $\left.P_{2}\right)$ is a $k$-rational point [0:1:0] (resp. [1:0:0]) of $E$. Moreover, $U_{1} \cap U_{2}$ is a product of $A_{k}^{1}$ and $E \backslash\left\{P_{1}, P_{2}\right\}$. Since $H_{\text {rig }}^{1}\left(U_{i} / K\right) \cong H_{\text {rig }}^{1}\left(E \backslash\left\{P_{i}\right\} / K\right) \cong H_{\text {rig }}^{1}(E)$ for $i=1,2$ and

$$
0 \rightarrow H_{\text {rig }}^{1}(E) \rightarrow H_{\text {rig }}^{1}\left(E \backslash\left\{P_{1}, P_{2}\right\} / K\right) \rightarrow K(-1) \rightarrow 0
$$

is exact by the Künneth formula [8, Théorème 3.2] and the excision sequence in Proposition 2.4, we have

$$
H_{\text {rig }}^{r}(U / K)=\left\{\begin{array}{cl}
K(0) & \text { if } r=0, \\
H_{\text {rig }}^{1}(E / K) & \text { if } r=1, \\
K(-1) & \text { if } r=2, \\
0 & \text { otherwise }
\end{array}\right.
$$

by the Čech spectral sequence associated to the covering above in Proposition 2.5. Using the Poincaré duality with respect to $U$ [8, Théorème 2.4] and the excision sequence of rigid cohomology with compact support, we have

$$
H_{\text {rig }}^{r}(X / K)=\left\{\begin{array}{cl}
K(0) & \text { if } r=0, \\
0 & \text { if } r=1, \\
K(-1) & \text { if } r=2, \\
H_{\text {rig }}^{1}(E / K)(-1) & \text { if } r=3, \\
K(-2) & \text { if } r=4, \\
0 & \text { otherwise. }
\end{array}\right.
$$

Hence the natural restriction map

$$
H_{\text {rig }}^{1}(X / K) \rightarrow H_{\text {rig }}^{1}(U / K)
$$

is not surjective. Since a nontrivial element of $H_{\text {rig }}^{1}(U / K)$ corresponds to an overconvergent connection on an extension of the unit object by the unit object by Theorem 2.13, the restriction functor $j_{U}^{\dagger}$ is not essentially surjective.

Let $\widetilde{g}: \widetilde{X} \rightarrow X$ be a desingularization obtained by a blowing up along the reduced closed point $Z$. Then the exceptional divisor $\widetilde{Z}=\widetilde{g}^{-1}(Z)$ is isomorphic to the elliptic curve $E$. Applying an excision sequence for $\widetilde{X}$ and 
$U=\widetilde{X} \backslash \widetilde{Z}$, we have

$$
H_{\text {rig }}^{r}(\widetilde{X} / K)=\left\{\begin{array}{cl}
K(0) & \text { if } r=0, \\
H_{\text {rig }}^{1}(E / K) & \text { if } r=1, \\
K(-1) \oplus K(-1) & \text { if } r=2, \\
H_{\text {rig }}^{1}(E / K)(-1) & \text { if } r=3, \\
K(-2) & \text { if } r=4, \\
0 & \text { otherwise }
\end{array}\right.
$$

by the weight reason. Indeed, $H_{\text {rig }}^{2}(X / K)(1)$ is generated by the cycle classes corresponding to $\widetilde{Z}$ and the strict transform $\widetilde{Z}^{\prime}\left(\cong \mathbb{P}_{k}^{1}\right)$ of $X \backslash U_{1}$. Hence the natural homomorphism $\widetilde{g}^{*}: H_{\text {rig }}^{1}(X / K) \rightarrow H_{\text {rig }}^{1}(\widetilde{X} / K)$ is not bijective.

In etale cases A. Grothendieck's purity, $\pi_{1}^{\text {alg }}(X \backslash Z) \stackrel{\cong}{\longrightarrow} \pi_{1}^{\text {alg }}(X)$, holds for a pair $(X, Z)$ consisting of a locally complete intersection $X$ and a closed subscheme $Z$ of codimension $\geq 3$ [17, $X$, Théorème 3.4]. The case of the second example does not satisfy the assumption of Grothendieck's purity theorem because $Z$ is of codimension 2 .

Acknowledgments. The author expresses his gratitude to Professor K. Kato, who asked me the injectivity of the restriction map in rigid cohomology groups of degree 1 . In the early first version of the paper the author studied normal varieties in our main theorem. However, Shiho pointed out that the normality is not stable by an arbitrary base change of base fields and the suitable condition is geometrically unibranch. The author thanks Shiho for valuable suggestions and conversations. The author also thanks the referee for reading the manuscript carefully and to suggesting an improvement of Theorem 1.1 (1).

\section{REFERENCES}

[1] M. Artin - A. Grothendieck - J. L. Verdier, Theorie des topos et cohomologie etale des schemas (SGA4), Lecture Notes in Math., 269, 270, and 305, Springer-Verlag, Heidelberg, 1972/3.

[2] F. Baldassarri - P. Berthelot, On Dwork cohomology for singular hypersurfaces, Geometric aspects of Dwork theory. Vol. I, II, pp. 177-244, Walter de Gruyter GmbH \& Co. KG, Berlin, 2004.

[3] F. BALdAssarri - B. ChiarellotTo, Algebraic versus rigid cohomology with logarithmic coefficients, Barsotti Symposium in Algebraic Geometry, Perspect. Math., 15, Academic press, San Diego, CA (1994), pp. 11-50. 
[4] V. G. Berkovich, Integration of one-forms on p-adic analytic spaces, Annals of Math. Studies 162, Princeton University Press, Princeton, NJ, 2007.

[5] P. Berthelot, Géométrie rigide et cohomologie des variétés algébriques de caractéristique $p$, Introductions aux cohomologies p-adiques (Luminy, 1984). Mém. Soc. Math. France (N.S.) No. 23, 3 (1986), pp. 7-32.

[6] P. Berthelot, Cohomologie rigide et cohomologie rigide à supports propres, Première partie, preprint, 1996.

[7] P. Berthelot, Finitude et pureté cohomologique en cohomologie rigide, With an appendix in English by Aise Johan de Jong. Invent. Math. 128, no. 2 (1997), pp. 329-377.

[8] P. Berthelot, Dualité de Poincaré et formule de Künneth en cohomologie rigide, C.R. Acad. Sc. Paris Sér. I Math. 325, no. 5 (1997), pp. 493-498.

[9] S. Bosch - U. GÜNTZER - R. REMmerT, Non-archimedean analysis, Grundlehren der Mathematischen Wissenschaften 261, Springer-Verlag, Berlin, 1984.

[10] D. CARo - N. TsuzuKI, Overholonomicity of overconvergent $F$-isocrystals over smooth varieties, Annals of Math. 176, no. 2 (2012), pp. 747-813.

[11] B. Chiarellotto - B. Le Stum, Sur la pureté de la cohomologie cristalline, C. R. Acad. Sci. Paris Sér. I Math. 326, no. 8 (1998), pp. 961-963.

[12] B. Chiarellotto - B. Le Stum, F-isocristaux unipotents, Compositio Math. 116, no. 8 (1999), pp. 81-110.

[13] B. Chiarellotto - N. Tsuzuki, Cohomological descent of rigid cohomology for étale coverings, Rend. Sem. Mat. Univ. Padova 109 (2003), pp. 63-215.

[14] B. ConraD, Deligne's note on Nagata compactifications, J. Ramanujan Math. Soc. 22, no. 3 (2007), pp. 205-257.

[15] R. ElkIK, Solutions d'équations à coefficients dans un anneau hensélien, Ann. Sci. École Norm. Sup. 6 (1973), pp. 553-603.

[16] A. GROTHENDIECK, Revétements étale et groupe fondemental (SGA1), Lecture Notes in Math. 224, Springer-Verlag, Heidelberg, 1971.

[17] A. GRothendeck, Cohomologie locale des faisceaux coherents et theoremes de Lefschetz locaux et globaux (SGA2), Augmenté d'un exposé par Michéle Raynaud. Séminaire de Géométrie Algébrique du Bois-Marie, 1962. Advanced Studies in Pure Mathematics, Vol. 2. North-Holland Publishing Co., Amsterdam; Masson \& Cie, Éditeur, Paris, 1968.

[18] A. Grothendieck - J. Dieudonné, Éléments de géométrie algébrique III, Étude cohomologique des faisceaux cohérents, Inst. Hautes Études Sci. Publ. Math. 11 (1961), pp. 5-167; 17 (1963), pp. 5-91.

[19] A. GRothendiECK - J. DiEudonné, Éléments de géométrie algébrique IV, Étude locale des schémas et des morphismes de schémas, Inst. Hautes Études Sci. Publ. Math. 20 (1964), pp. 5-259; 24 (1965), pp. 5-231; 32 (1967), pp. 5-361.

[20] A. J. DE Jong, Smoothness, semi-stability and alterations, Inst. Hautes Études Sci. Publ. Math. 83 (1996), pp. 51-93.

[21] K. Kedlaya, Semistable reduction for overconvergent F-isocrystals, I: Unipotence and logarithmic extensions, Compositio Math. 143, no. 5 (2007), pp. 1164-1212.

[22] M. NAGATA, Imbedding of an abstract variety in a complete variety, J. Math. Kyoto, 2 (1962), pp. 1-10.

[23] M. NAGATA, A generalization of the imbedding problem of an abstract variety in a complete variety, J. Math. Kyoto Univ. 3 (1963), pp. 89-102. 
[24] M. Nagata, Field theory, Monographs and textbooks in pure and applied mathematics 40, Marcel Dekker Inc. New York and Basel, 1977.

[25] Y. NAKKAJIMA, Weight filtration and slope filtration on the rigid cohomology of a variety in characteristic $p>0$, to appear in Mém. Soc. Math. France.

[26] A. OGus, F-isocrystals and de Rham cohomology II - convergent isocrystals, Duke Math. J. 51, no. 4 (1984), pp. 765-850.

[27] A. SHiнo, Crystalline fundamental groups. II. Log convergent cohomology and rigid cohomology, J. Math. Sci. Univ. Tokyo, 9, no. 1, (2002), pp. 1-163.

[28] A. SHIHo, Relative log convergent cohomology and relative rigid cohomology I, preprint, 2007. http://arxiv.org/abs/0707.1742v2

[29] A. SHIHO, Relative log convergent cohomology and relative rigid cohomology II, preprint, 2007. http://arxiv.org/abs/0707.1743v2

[30] N. TsuzukI, On the Gysin isomorphism of rigid cohomology, Hiroshima Math. J. 29, no. 3 (1999), pp. 479-527.

[31] N. TsuzukI, Morphisms of F-isocrystals and the finite monodromy theorem for unit-root F-isocrystals, Duke Math. J. 111, no. 3 (2002), pp. 385-418.

[32] N. Tsuzuki, Cohomological descent of rigid cohomology for proper coverings, Invent. Math. 151, no. 1 (2003), pp. 101-133.

[33] N. Tsuzuki, Cohomological descent in rigid cohomology, Geometric aspects of Dwork theory. Vol. I, II, pp. 931-981, Walter de Gruyter GmbH \& Co. KG, Berlin, 2004.

Manoscritto pervenuto in redazione 15 Novembre 2011. 
